\title{
Direct numerical simulations of supercritical fluid mixing layers applied to heptane-nitrogen
}

\author{
By RICHARD S. MILLER†, KENNETH G. HARSTAD \\ AND JOSETTE BELLAN
}

Jet Propulsion Laboratory, California Institute of Technology, Pasadena, CA 91109-8099, USA

(Received 15 July 1999 and in revised form 16 November 2000)

Direct numerical simulations (DNS) are conducted of a model hydrocarbon-nitrogen mixing layer under supercritical conditions. The temporally developing mixing layer configuration is studied using heptane and nitrogen supercritical fluid streams at a pressure of $60 \mathrm{~atm}$ as a model system related to practical hydrocarbon-fuel/air systems. An entirely self-consistent cubic Peng-Robinson equation of state is used to describe all thermodynamic mixture variables, including the pressure, internal energy, enthalpy, heat capacity, and speed of sound along with additional terms associated with the generalized heat and mass transport vectors. The Peng-Robinson formulation is based on pure-species reference states accurate to better than $1 \%$ relative error through comparisons with highly accurate state equations over the range of variables used in this study $(600 \leqslant T \leqslant 1100 \mathrm{~K}, 40 \leqslant p \leqslant 80 \mathrm{~atm})$ and is augmented by an accurate curve fit to the internal energy so as not to require iterative solutions. The DNS results of two-dimensional and three-dimensional layers elucidate the unique thermodynamic and mixing features associated with supercritical conditions. Departures from the perfect gas and ideal mixture conditions are quantified by the compression factor and by the mass diffusion factor, both of which show reductions from the unity value. It is found that the qualitative aspects of the mixing layer may be different according to the specification of the thermal diffusion factors whose value is generally unknown, and the reason for this difference is identified by examining the second-order statistics: the constant Bearman-Kirkwood (BK) thermal diffusion factor excites fluctuations that the constant Irwing-Kirkwood (IK) one does not, and thus enhances overall mixing. Combined with the effect of the mass diffusion factor, constant positive large BK thermal diffusion factors retard diffusional mixing, whereas constant moderate IK factors tend to promote diffusional mixing. Constant positive BK thermal diffusion factors also tend to maintain density gradients, with resulting greater shear and vorticity. These conclusions about IK and BK thermal diffusion factors are speciespair dependent, and therefore are not necessarily universal. Increasing the temperature of the lower stream to approach that of the higher stream results in increased layer growth as measured by the momentum thickness. The three-dimensional mixing layer exhibits slow formation of turbulent small scales, and transition to turbulence does not occur even for a relatively long non-dimensional time when compared to a previous, atmospheric conditions study. The primary reason for this delay is the initial density stratification of the flow, while the formation of strong density gradient regions both in the braid and between-the-braid planes may constitute a secondary reason for the hindering of transition through damping of emerging turbulent eddies.

$\dagger$ Present address: Department of Mechanical Engineering, Clemson University, South Carolina

$\ddagger$ Author for correspondence: Josette.Bellan@jpl.nasa.gov 


\section{Introduction}

Mixing layers naturally occur when two fluids move with a relative parallel velocity, but the characteristics of these layers also depend upon other parameters such as the density ratio (Dimotakis 1986) and the particular form of the perturbations or lack of perturbations present in the flow (Cortesi, Yadigaroglu \& Banerjee 1998; Cortesi et al. 1999). Numerous theoretical studies exist of gaseous mixing layers at standard (atmospheric) pressure and temperature conditions (Gerz, Schumann \& Elghobashi 1989; Cortesi et al. 1998, 1999, etc.), these being the conditions prevailing in most experiments that theoretical investigations seek to emulate. However, there is an entire class of mixing layers that has received much less attention owing to the lack of guiding theoretical and/or experimental studies: supercritical mixing layers. Such mixing layers occur in nature in planetary atmospheres (e.g. Jupiter), and in machines such as combustion engines (diesel and gas turbine) where the fuel is introduced in a jet-like manner in a high pressure and temperature environment. This last example is essentially different from the process of atomization wherein the fluid in the jet is liquid and the shear layer evolving at its surface creates a rollup (Kelvin-Helmholtz instability) and eventual breakup of the jet into small, irregularly shaped entities (ligaments) and drops. When the jet is introduced into an environment at a pressure, $p$, or temperature, $T$, higher than that of the critical point of the substance, the fluid is no longer a liquid (Hirshfelder, Curtis \& Bird 1964; Prausnitz, Lichtenthaler \& de Azevedo 1986) and does not behave according to the well-established atmospheric observations (Mayer et al. 1996, 1998 and Chehroudi, Talley \& Coy 1999). For example, as the pressure of the environment is raised, the jet not only changes its appearance to increasingly resemble that of a gaseous jet, but more importantly there is no evidence of drop formation as shown by Cheroudi et al. (1999). Instead, threador finger-like entities emerge from the jet, which rapidly dissolve away from the jet core (Chehroudi et al. 1999). With increasing pressure, the observations also show a decrease in the length and the thickness of the internal core. These observations have direct relevance to hydrocarbon injection in diesel and gas turbine engines because the critical pressure of most fuel hydrocarbons is in the range of $1.5-3 \mathrm{MPa}$ and the maximum pressure attained in these engines is about $6 \mathrm{MPa}$. Therefore, because the critical point of a mixture depends on its composition, it is clear that the fuel may be subjected to both subcritical and supercritical conditions.

Fluid breakup under supercritical conditions has only been studied empirically (Mayer et al. 1996, 1998 and Chehroudi et al. 1999), and there is almost no information on the detailed physical processes governing it. The theoretical study of Oefelein \& Yang (1996), although not specifically addressing fluid breakup under supercritical conditions, represents a unique exception to the lack of turbulent supercritical flow simulations. In that study Oefelein \& Yang (1996) investigate transcritical sprays using large eddy simulations by employing a Smagorinsky model with a constant whose value is derived from single-phase-flow compressible turbulence investigations. It is not clear how this value of the Smagorinsky constant applies to the situation studied by Oefelein \& Yang (1996), and how it is consistent with their considerations of drag forces on the drops (i.e. either there is a two-phase flow where drag is a valid concept, but the Smagorinsky constant may be incorrect; or there is a singlephase flow with the Smagorinsky constant being relevant, but then drag is no longer a relevant concept). Moreover, Oefelein \& Yang (1996) neglect Soret and Dufour effects without providing an estimate of the validity of this assumption. The present study constitutes a first step towards bridging the knowledge gap between the needed and existing information regarding Soret and Dufour effects in the context of supercritical 
mixing layers. Since accurate, detailed information on Soret and Dufour coefficients in high-pressure flows is lacking, in this paper we perform a parametric study by using a set of constant thermal diffusion factor values. We address in this investigation the detailed processes that may lead to the formation of small scales in the shear layer of a fluid jet through direct numerical simulation (DNS) of a mixing layer. (Although mixing layers are different from jets, the jet mixing region behaves essentially as a mixing layer.) Because for varying $(p, T)$ the critical locus for a mixture of $N$ substances is usually a complicated $N-1$ manifold, the 'supercritical' terminology throughout this paper which discusses hydrocarbon-nitrogen mixing refers to the conditions with respect to the pure hydrocarbon.

Since there are common aspects to fluid jets and mixing layers, investigations of density-stratified mixing layers are pertinent to the study of supercritical fluid jet disintegration. An isolated study of density stratification effects in spatially evolving high-pressure mixing layers is that of Brown \& Roshko (1974). Using a pressurized (up to $10 \mathrm{~atm}$ ) experimental facility the authors compared the spreading angle from a nitrogen-air mixing layer at $7 \mathrm{~atm}$ where the density ratio was considered to be close to unity, to that of a nitrogen-helium flow in $4 \mathrm{~atm}$ where the density ratio was changed by switching the location of nitrogen from top to bottom. The stratification effects on the development of the mixing layer were only modest: a large (factor of 7) increase in $\rho_{2} / \rho_{1}$ (the subscripts 1 and 2 refer to the upper and lower streams, respectively) resulted only in a factor of 2 decrease in the spreading angle.

This paper is organized as follows: The conservation equations are presented in $\S 2$ where we also include the specifics of the equation of state and transport coefficients. These conservation equations are based upon those previously presented for a multicomponent mixture by Harstad \& Bellan (1998), and validated for a heptane fluid drop in nitrogen by Harstad \& Bellan (2000). As discussed by Harstad \& Bellan (1998) and Harstad \& Bellan (2000), the most general formulation for the diffusional heat and mass fluxes is that of fluctuation-dissipation theory formulated by Keizer (1987). This theory is not only entirely consistent with non-equilibrium thermodynamics, but also allows the definition of the relationships between fluxes and forces for a general fluid which continuum theory does not give; it is customary within the continuum formulation to extend kinetic theory of rarified gases to describe more general cases as in Chapman \& Cowling (1970). In $\S 3$ we describe the configuration, the initial conditions, and briefly mention the numerical approach, which has been discussed in detail elsewhere (Miller \& Bellan 1999). Section 4 is devoted to the discussion of results, emphasizing the peculiarities introduced by the high-pressure thermodynamics; particular attention is given to the influence of the thermal diffusion factors (Chapman \& Cowling 1970) which are the additional transport coefficients associated with Soret and Dufour effects that may become important at high pressure. Following the validated fluid model, two definitions of the thermal diffusion factor appear in the formulation, each being related to a mathematical form of the heat flux vector. While only one of these definitions converges to the kinetic theory value in the low-pressure limit, both of these forms of the thermal diffusion factor could be measured in appropriate experiments; however, such data are generally lacking. Therefore, although an approximate pressure and temperature variation of the thermal diffusion factor has been identified for heptane-nitrogen during the previously conducted model validation, the effect of the identity and of the imposed constant value of the thermal diffusion factor is first investigated by studying two-dimensional mixing layers. Using the knowledge of the thermal diffusion factor obtained from the validated model of Harstad \& Bellan (2000), a single three-dimensional simulation is 
performed and its results are discussed. In particular, the relevance of specific visual features exhibited by visualizations of the simulations to the evolution of turbulence scales is highlighted. Conclusions are presented in $\S 5$ along with further discussion regarding knowledge needs related to the values of the thermal diffusion factors.

\section{Mathematical formulation}

The compressible form of the conservation equations for a binary mixture of general (Newtonian) fluids are

$$
\begin{gathered}
\frac{\partial \rho}{\partial t}+\frac{\partial}{\partial x_{j}}\left[\rho u_{j}\right]=0, \\
\frac{\partial}{\partial t}\left(\rho u_{i}\right)+\frac{\partial}{\partial x_{j}}\left[\rho u_{i} u_{j}+p \delta_{i j}-\tau_{i j}\right]=0, \\
\frac{\partial}{\partial t}\left(\rho e_{t}\right)+\frac{\partial}{\partial x_{j}}\left[\left(\rho e_{t}+p\right) u_{j}-u_{i} \tau_{i j}+q_{I K, j}\right]=0, \\
\frac{\partial}{\partial t}\left(\rho Y_{h}\right)+\frac{\partial}{\partial x_{j}}\left[\rho Y_{h} u_{j}+J_{h j}\right]=0,
\end{gathered}
$$

where $\rho$ is the density, $u_{i}$ is the velocity, $e_{t}=e+u_{i} u_{i} / 2$ is the total energy (i.e. internal energy, $e$, plus kinetic energy), $p$ is the thermodynamic pressure (the temperature is $T$ ) and $Y_{h}$ is the mass fraction of heptane (the mass fraction of nitrogen is $Y_{n}=1-Y_{h}$ ). Furthermore, $\boldsymbol{q}_{I K}$ is the Irwing-Kirkwood (subscript $I K$ ) form of the heat flux vector (see Sarman \& Evans 1992 and discussion below), $\boldsymbol{J}_{h}$ is the heptane mass flux vector and $\tau_{i j}$ is the Newtonian viscous stress tensor:

$$
\tau_{i j}=\mu\left[\frac{\partial u_{i}}{\partial x_{j}}+\frac{\partial u_{j}}{\partial x_{i}}-\frac{2}{3} \frac{\partial u_{k}}{\partial x_{k}} \delta_{i j}\right],
$$

where $\delta_{i j}$ is the Kronecker delta function, and $\mu$ is the mixture viscosity which is in general a function of the thermodynamic state variables. For an isotropic fluid, $\tau_{i j}$ usually contains terms proportional to both shear and bulk viscosity; however, the Keizer (1987) fluctuation-dissipation theory (see details below) clearly distinguishes between these two contributions: whereas shear viscosity arises from molecular interactions transferring momentum between neighbouring fluid elements, bulk viscosity is related to changes, or different states, of internal energy. Since bulk viscosity effects generally tend to be small (see Bird, Stewart \& Lightfoot 1960), here they are neglected; the value of the bulk viscosity is uncertain or unknown even under the best circumstances.

The governing equations for the mixing layer comprise (2.1)-(2.5) and the equation of state whose form is discussed in $\S 2.1$. To solve the system of governing equations one must specify the mathematical forms of $\boldsymbol{q}_{I K}$ and $\boldsymbol{J}_{h}$ as functions of the dependent variables and of the transport coefficients, and give boundary and initial conditions for the mixing layer; these are presented in $\$ \S 2.2,2.3$ and 3, respectively. The calculation of the transport coefficients other than the thermal diffusion factors (see below) is discussed in $\S 2.4$.

\subsection{Equation of state}

It is well known that the perfect gas relationship is a good representation of the equation of state (EOS) only at standard conditions (Prausnitz et al. 1986); as the 
pressure and temperature increase, departures from the perfect gas behaviour occur whose severity depends upon the species. Thermodynamics mitigates this situation through the microscopic corresponding states theory (Prausnitz et al. 1986) whose main result is the fact that the EOS is a universal function relating the appropriately non-dimensionalized (using molecular parameters) pressure, temperature and molar volume. Traditional EOSs such as the Redlich-Kwong, the Soave-Redlich-Kwong and the Peng-Robinson (PR) discussed by Prausnitz et al. (1986) have indeed been shown to be good representations of fluid behaviour at large values of the 'reduced' conditions (subscript $r$ ) defined by $p_{r}=p / p_{c}$ and $T_{r}=T / T_{c}$ where the subscript $c$ denotes the critical thermodynamic state. More recently, Harstad, Miller \& Bellan (1997) have presented computationally efficient forms of EOSs and have also shown that it is possible to extend their validity beyond the range of data by using thermodynamic departure functions. The usefulness of all these EOSs extends to multicomponent mixtures since 'mixing rules' (see Prausnitz et al. 1986) allow in fact the calculation of the EOS coefficients for a wide range of species combinations.

In this study we choose to represent the binary mixture by the PR EOS because it is computationally one of the simplest, and because the pure-species reference states were found to be accurate to better than $1 \%$ relative error through comparisons with the accurate state equations of Harstad et al. (1997) over the range of variables used in this study. The cubic PR EOS is

$$
p=R_{u} T /\left(v-B_{m}\right)-A_{m} /\left(v^{2}+2 v B_{m}-B_{m}^{2}\right),
$$

and the two (mixture) parameters specifying the equation are

$$
A_{m}=\sum_{\alpha} \sum_{\beta} X_{\alpha} X_{\beta} A_{\alpha \beta}, \quad B_{m}=\sum_{\alpha} X_{\alpha} B_{\alpha} .
$$

In the above equations, the subscripts $\alpha$ and $\beta$ denote the individual species and take values of $n$ and $h$ (no summation over Greek indices), the summations indicate summing over both species, $R_{u}$ is the universal gas constant, the mole fraction $X$ is related to the mass fraction by $m_{\alpha} X_{\alpha}=m Y_{\alpha}$ where $m_{\alpha}$ is the molecular weight of pure species $\alpha$ and the mixture molecular weight is $m=X_{n} m_{n}+X_{h} m_{h}$. Furthermore, the molar volume $v$ is related to the density by $v=m / \rho$. The components of the PR parameters are provided by an appropriate set of mixing rules which vary for different state equations and may even have variations for the same state equation; in the present model we follow the mixing rules given in Harstad et al. (1997), and the exact set of mixing rules is provided in Appendix A. These mixing rules are a synthesis of those in Reid, Prausnitz \& Polling (1987) and Prausnitz et al. (1986).

To ensure self-consistency in the model, all of the thermodynamic properties of the flow are here calculated from the same equation of state. The properties of interest for the present fluid dynamics simulations are the molar enthalpy $(h)$, the constant-pressure molar heat capacity $\left(C_{p}\right)$ and the speed of sound $\left(a_{s}\right)$. Each of these properties can be obtained through various derivatives and functions of the Gibbs energy $(G)$ :

$$
G\left(T, p, X_{\alpha}\right)=\int_{v}^{v_{u}} p\left(v^{\prime}, T, X_{\alpha}\right) \mathrm{d} v^{\prime}+p v-R_{u} T+\sum_{\alpha} X_{\alpha}\left[G_{\alpha}^{0}+R_{u} T \ln \left(X_{\alpha}\right)\right],
$$

where the superscript 0 represents the 'low-pressure' reference condition for the integration as generally used in the departure function formalism described by Prausnitz et al. (1986), and $v_{u}$ is given below. Note that the integral is ill defined for a zero- 
pressure reference condition; hereinafter we choose $p^{0}=1$ bar such that $v_{u}=R_{u} T / p^{0}$. From this relation we then obtain

$$
\begin{gathered}
h=G-T\left(\frac{\partial G}{\partial T}\right)_{p, X}=h^{0}+p v-R_{u} T+K_{1}\left(A_{m}-T \frac{\partial A_{m}}{\partial T}\right), \\
C_{p}=\left(\frac{\partial h}{\partial T}\right)_{p, X}=C_{p}^{0}-T \frac{(\partial p / \partial T)_{v, X}^{2}}{(\partial p / \partial v)_{T, X}}-R_{u}-T \frac{\partial^{2} A_{m}}{\partial T^{2}} K_{1},
\end{gathered}
$$

where the partial derivatives of $A_{m}$ are given in Appendix B, $h$ is the molar enthalpy, $h^{0}$ is the reference molar enthalpy, $C_{p}$ is the molar heat capacity, $C_{p}^{0}=\partial h^{0} / \partial T$ is the reference molar heat capacity,

$$
\begin{gathered}
\left(\frac{\partial p}{\partial T}\right)_{v, X}=R_{u} /\left(v-B_{m}\right)-\left(\partial A_{m} / \partial T\right) /\left(v^{2}+2 v B_{m}-B_{m}^{2}\right), \\
\left(\frac{\partial p}{\partial v}\right)_{T, X}=\frac{-R_{u} T}{\left(v-B_{m}\right)^{2}}\left[1-2 A_{m}\left\{R_{u} T\left(v+B_{m}\right)\left(\frac{v}{v-B_{m}}+\frac{B_{m}}{v+B_{m}}\right)^{2}\right\}^{-1}\right], \\
K_{1}=\frac{1}{2 \sqrt{2} B_{m}} \ln \left[\frac{v+(1-\sqrt{2}) B_{m}}{v+(1+\sqrt{2}) B_{m}}\right] .
\end{gathered}
$$

Finally, for real fluids the speed of sound

$$
a_{s}=\sqrt{\frac{1}{\rho \kappa_{s}}}
$$

is given in terms of the isentropic compressibility

$$
\kappa_{s}=\kappa_{T}-v T \alpha_{v}^{2} / C_{p},
$$

which is related to the expansivity $\left(\alpha_{v}\right)$ and the isothermal compressibility $\left(\kappa_{T}\right)$, which can be expressed by:

$$
\alpha_{v}=-\frac{(\partial p / \partial T)_{v, X}}{v(\partial p / \partial v)_{T, X}}, \quad \kappa_{T}=\frac{-1}{v(\partial p / \partial v)_{T, X}} .
$$

These equations specify the entire thermodynamics of the binary mixture.

\subsection{Heat and mass transport}

Although similar equations, as published by others, have been derived in textbooks, the originality of the Harstad \& Bellan (2000) derivation is to distinguish between thermal diffusion factors associated with different forms of the heat flux (see below), and define the thermal conductivity from the transport matrix (see below) in terms of these thermal diffusion factors. The distinction between the two thermal diffusion factors was not incidental; in fact, it was crucial in enabling validation of the model with microgravity data. The model presented here is based upon that of Harstad \& Bellan (2000).

Within this general theory, the diffusional fluxes are linear combinations of terms proportional to the gradients of the temperature, of the molar fractions and of the pressure, and the proportionality constants are the elements of the 'transport matrix'. 
One property of the transport matrix is that its elements are symmetric as they satisfy the Onsager reciprocal relationships. According to Harstad \& Bellan (1998, 2000), the form of the diffusional fluxes is

$$
\begin{gathered}
q_{I K, j}=-\left[\lambda_{I K}^{\prime} \frac{\partial T}{\partial x_{j}}+\alpha_{I K} R_{u} T\left(\frac{m}{m_{n} m_{h}}\right) J_{h j}^{\prime}\right], \\
J_{h j}=-\left[J_{h j}^{\prime}+\alpha_{B K} Y_{n} Y_{h} \rho D / T \frac{\partial T}{\partial x_{j}}\right], \\
J_{h j}^{\prime}=\rho D\left[\alpha_{D} \frac{\partial Y_{h}}{\partial x_{j}}+\frac{Y_{n} Y_{h}}{R_{u} T}\left(\frac{m_{n} m_{h}}{m}\right)\left(\frac{v_{, h}}{m_{h}}-\frac{v_{, n}}{m_{n}}\right) \frac{\partial p}{\partial x_{j}}\right],
\end{gathered}
$$

where (2.17) is the Irwing-Kirkwood form of the heat flux (Sarman \& Evans 1992), the binary diffusion coefficient is $D$, the mass diffusion factor is $\alpha_{D}$, and the thermal conductivity $\lambda_{I K}^{\prime}$ is defined by Harstad \& Bellan (2000) from the transport matrix. It can be shown that $\lambda_{I K}^{\prime}$ does not correspond to the kinetic theory (subscript $K T$ ) definition of the thermal conductivity in that $\lim _{p \rightarrow 0} \lambda_{I K}^{\prime} \neq \lambda_{K T}$ but it is related to the thermal conductivity, $\lambda$, through

$$
\lambda_{I K}^{\prime}=\lambda+X_{n} X_{h} \alpha_{I K} \alpha_{B K} R_{u} \rho D / m,
$$

where $\lim _{p \rightarrow 0} \lambda=\lambda_{K T}$ as discussed in Harstad \& Bellan (2000). In the above equations $\alpha_{I K}$ and $\alpha_{B K}$ are the thermal diffusion factors corresponding to the IK and the Bearman-Kirkwood (subscript BK) forms of the heat flux (Sarman \& Evans 1992), respectively; they are the new transport coefficients that are introduced by the Soret (in the molar fluxes) and the Dufour (in the heat flux) terms of the transport matrix, and are characteristic of the particular species pairs under consideration. Properties of these thermal diffusion factors are that $\lim _{p \rightarrow 0} \alpha_{I K} \neq \alpha_{K T}$ and $\lim _{p \rightarrow 0} \alpha_{B K}=\alpha_{K T}$. Currently there is no information as to the functional form of $\alpha_{I K}$ and $\alpha_{B K}$ with respect to the primary variables $\left(p, T, Y_{i}\right)$ and/or their magnitudes, but it can be shown that they are related through a relationship derived in Harstad \& Bellan (2000)

$$
\alpha_{I K}=\alpha_{B K}+\frac{1}{R_{u} T}\left(\frac{m_{n} m_{h}}{m}\right)\left(\frac{h_{, h}}{m_{h}}-\frac{h_{, n}}{m_{n}}\right) .
$$

We discuss in the Results section, $\S 4$, the effect of the identity (IK or BK) and the magnitude of these thermal diffusion coefficients.

Thermodynamic related quantities appearing in (2.19) and (2.21) are the partial molar volumes $v_{, \alpha}=\partial v / \partial X_{\alpha}$ and the partial molar enthalpies $h_{, \alpha}=\partial h / \partial X_{\alpha}$ :

$$
\begin{gathered}
v_{, \alpha}=\frac{-1}{(\partial p / \partial v)_{T, X}}\left[\frac{R_{u} T}{v-B_{m}}+\frac{R_{u} T B_{\alpha}}{\left(v-B_{m}\right)^{2}}+\frac{2 A_{m}\left(v-B_{m}\right) B_{\alpha}}{\left(v^{2}+2 v B_{m}-B_{m}^{2}\right)^{2}}-\frac{2 \sum_{\beta} A_{\alpha \beta} X_{\beta}}{v^{2}+2 v B_{m}-B_{m}^{2}}\right], \\
h_{, \alpha}=h_{\alpha}^{0}+p v_{, \alpha}-R_{u} T+\left(A_{m}-T \frac{\partial A_{m}}{\partial T}\right) \frac{v_{, \alpha}-v B_{\alpha} / B_{m}}{v^{2}+2 v B_{m}-B_{m}^{2}} \\
+K_{1}\left[\frac{\partial A_{m}}{\partial X_{\alpha}}-T \frac{\partial^{2} A_{m}}{\partial X_{\alpha} \partial T}-\left(A_{m}-T \frac{\partial A_{m}}{\partial T}\right) \frac{B_{\alpha}}{B_{m}}\right]
\end{gathered}
$$

where $v=X_{n} v_{, n}+X_{h} v_{, h}$ and $h=X_{n} h_{, n}+X_{h} h_{, h}$. The mass diffusion factor $\alpha_{D}$ is also a 


\begin{tabular}{ccccccc} 
Species & $m, \frac{\mathrm{kg}}{\mathrm{kg} \mathrm{mole}}$ & $T_{c}, \mathrm{~K}$ & $p_{c}$, atm & $v_{c}, \frac{\mathrm{m}^{3}}{\mathrm{~kg} \mathrm{~mole}}$ & $Z_{c}$ & $\Omega$ \\
Nitrogen & 28.013 & 126.26 & 33.55 & 0.0898 & 0.290 & 0.039 \\
Heptane & 100.205 & 540.3 & 27.04 & 0.432 & 0.263 & 0.349 \\
\multicolumn{7}{c}{ TABLE 1 1. Pure-species properties. }
\end{tabular}

thermodynamic function which is calculated from the fugacity coefficients, $\varphi_{\alpha}$ (which are related to the Gibbs energy), as follows:

$$
\alpha_{D}=1+X_{\alpha} \frac{\partial \ln \left(\varphi_{\alpha}\right)}{\partial X_{\alpha}} .
$$

Note that $\alpha_{D}$ is independent of which species is chosen in the evaluation. After significant manipulation we obtain the explicit relationship

$$
\begin{aligned}
\alpha_{D}=1+X_{n} X_{h} & \left\{\frac{R_{u} T}{(\partial p / \partial v)_{T, X}}\left(\frac{1}{B_{m}^{2}}\right)\right. \\
& \left.\times\left[\frac{B_{h}-B_{n}}{v-B_{m}}+\frac{B_{n} \partial A_{m} / \partial X_{h}-B_{h} \partial A_{m} / \partial X_{n}}{R_{u} T\left(v^{2}+2 v B_{m}-B_{m}^{2}\right)}\right]^{2}+K_{2}\right\},
\end{aligned}
$$

where

$$
K_{2}=\left(\frac{B_{h}-B_{n}}{B_{m}}\right)^{2}+\frac{2}{R_{u} T}\left(\frac{K_{1}}{B_{m}^{2}}\right)\left(A_{h h} B_{n}^{2}+A_{n n} B_{h}^{2}-2 A_{n h} B_{n} B_{h}\right) .
$$

The formulation is now closed with the exception of specifying the transport coefficients $\mu, \lambda, D$ and $\alpha_{I K}$ (or $\alpha_{B K}$ ) and also the molar reference enthalpy of the mixture, $h^{0}$.

\subsection{Transport coefficients and reference enthalpy}

In the context of DNS it is futile to attempt the use of exact values of the transport coefficients along with their thermodynamic dependences since it would be impossible to resolve the length scales associated with the resulting Reynolds numbers. To focus our discussion on transport coefficients, we now devote attention to the properties of both heptane and nitrogen which are shown in table 1. Even in the case of an arbitrarily small physical domain, the Schmidt number for the liquid-like fluid heptane is too large to resolve the species fields for interesting Reynolds numbers. We therefore limit the range of thermodynamic state space which will be considered to the range $600 \leqslant T \leqslant 1100 \mathrm{~K}, 40 \leqslant p \leqslant 80 \mathrm{~atm}$, and of course $0 \leqslant Y_{h} \leqslant 1$. This particular region is chosen for two primary reasons: (1) The PR EOS was found to be highly accurate within this region when compared to the more accurate model of Harstad et al. (1997) with the relative error being in no case greater than approximately $1 \%$ for both the entropy and enthalpy predictions (this error can be larger than $25 \%$ at $p=6 \mathrm{MPa}$ and $T=350 \mathrm{~K}$ ). (2) Contour plots (not shown) of the viscosity, and of the Schmidt $(\mathrm{Sc})$ and Prandtl $(\mathrm{Pr})$ numbers based on accurate species transport properties calculated as in Harstad \& Bellan (1998), revealed that the viscosity is predominantly a function of $T$ alone, whereas $S c$ and $P r$ are predominantly functions of the mass 
fraction. This leads to the relatively simplified diffusion coefficients

$$
\begin{gathered}
\mu=\mu_{R}\left(\frac{T}{\left(T_{1}+T_{2}\right) / 2}\right)^{0.7}, \quad T \text { in } \mathrm{K}, \\
S c=\frac{\mu}{\rho \alpha_{D} D}=1.5-Y_{h}, \quad \operatorname{Pr}=\frac{\mu C_{p} / m}{\lambda}=\frac{S c}{2 \exp \left(-3 Y_{h} / 2\right)},
\end{gathered}
$$

where $\mu_{R}$ is a reference viscosity and the reference temperatures $T_{1}$ and $T_{2}$ correspond to the free-stream temperatures for mixing layer simulations. These relations give qualitatively correct trends in that the Schmidt number is respectively greater than or less than unity for the proper mass fractions; however, the maximum value is 1.5 rather than 2 as observed in the more complete model. This allows the consideration of Reynolds numbers that have the same magnitude as those for which transition to turbulence was obtained in previous studies for both gas and drop-laden mixing layers (Miller \& Bellan 2000) making a comparison with different types of mixing layers possible.

The functional dependences of the thermal diffusion factors on the primary variables $\left(p, T, Y_{i}\right)$ are poorly understood, particularly at high pressure (see Bird et al. 1960 and Chapman \& Cowling 1970). For low-pressure conditions some theory exists suggesting that $\alpha_{B K}$ is nearly independent of the mass fraction (though it can generally be a function of T) (Bird et al. 1960 and Chapman \& Cowling 1970). Most calculations of thermal diffusion factors are performed using molecular dynamics theory, such as in Sarman \& Evans (1992); these calculations are very computationally-intensive and have generally been used for esoteric species or combinations which are easier to simulate. On the other hand, measurements of thermal diffusion factors are difficult in that they may be plagued by parasitic convective effects in experiments that are supposed to be of purely diffusive character, and suffer from dubious interpretation of data when these parasitic effects are not included in the equations used to determine the values of the thermal diffusion factors.

Finally, comparisons with the more accurate EOS of Harstad et al. (1997) show that the (low-pressure) mixture reference enthalpy

$$
h^{0}=X_{n} h_{n}^{0}+X_{h} h_{h}^{0},
$$

is well fit using

$$
\frac{h_{n}^{0}}{m_{n}}=656.72\left(T^{1.071}\right) \frac{\mathrm{J}}{\mathrm{kg} \mathrm{K}}, \quad \frac{h_{h}^{0}}{m_{h}}=27.877\left(T^{1.6414}\right) \frac{\mathrm{J}}{\mathrm{kg} \mathrm{K}} .
$$

\subsection{Internal energy curve fit}

The set of equations described above is closed and a solution can now be obtained; however, there is a choice to be made regarding the most convenient primitive variables. For example, in compressible flow simulations, the density, momentum and energy equations, and the above state equation must be solved iteratively to get both the temperature and pressure. In order to avoid computationally intensive iterations, a highly accurate fit was here obtained for the specific internal energy of the mixture (e) over the entire state space of interest, which is explicit in the temperature

$$
T=\left[\left(T_{L}\right)^{\theta}+\left(\frac{e-e_{L}}{e_{U}-e_{L}}\right)\left\{\left(T_{U}\right)^{\theta}-\left(T_{L}\right)^{\theta}\right\}\right]^{1 / \theta},
$$




$\begin{array}{ccccc}\text { Energy, } \mathrm{MJ} \mathrm{kg}{ }^{-1} & E_{0} & E_{1} & E_{2} & E_{3} \\ e_{1}\left(Y_{h} ; T_{L}, \rho_{L}\right) & 0.44142 & 0.51813 & -1.8118 \times 10^{-3} & 6.12810 \times 10^{-6} \\ e_{2}\left(Y_{h} ; T_{L}, \rho_{U}\right) & 0.41489 & 0.47173 & -4.4262 \times 10^{-2} & 2.25095 \times 10^{-3} \\ e_{3}\left(Y_{h} ; T_{U}, \rho_{L}\right) & 0.86090 & 1.78435 & -1.9375 \times 10^{-3} & 4.78990 \times 10^{-6} \\ e_{4}\left(Y_{h} ; T_{U}, \rho_{U}\right) & 0.85262 & 1.76317 & -4.9197 \times 10^{-2} & 2.59000 \times 10^{-3}\end{array}$

TABLE 2. Polynomial curve-fit coefficients.

where the subscripts $L$ and $U$ refer to the lower and upper limit of validity of the fit,

$$
\begin{gathered}
\theta=1.14+0.667 Y_{h}^{0.676}, \\
e_{L}=e_{1}+\left[\frac{\rho-\rho_{L}}{\rho_{U}-\rho_{L}}\right]\left(e_{2}-e_{1}\right), \\
e_{U}=e_{3}+\left[\frac{\rho-\rho_{L}}{\rho_{U}-\rho_{L}}\right]\left(e_{4}-e_{3}\right),
\end{gathered}
$$

and the upper and lower bounds are $\rho_{L}=8, \rho_{U}=248, T_{L}=600$ and $T_{U}=1100$ with the density in $\mathrm{kg} \mathrm{m}^{-3}$ and the temperature in $\mathrm{K}$. The limits of the density correspond to the pressure limits of 40 and $80 \mathrm{~atm}$. Finally, third-order polynomial fits were obtained for the four remaining internal energy functions $e_{1}\left(Y_{h} ; T_{L}, \rho_{L}\right)$, $e_{2}\left(Y_{h} ; T_{L}, \rho_{U}\right), e_{3}\left(Y_{h} ; T_{U}, \rho_{L}\right)$ and $e_{4}\left(Y_{h} ; T_{U}, \rho_{U}\right)$ as follows:

$$
e_{k}=E_{0}+E_{1} Y_{h}+E_{2} Y_{h}^{2}+E_{3} Y_{h}^{3}, \quad k=1,2,3,4,
$$

where the coefficients are provided in table 2. This particular fit also achieves better than $1 \%$ maximum relative error when compared to the PR internal energy $(e=h-p v)$.

\section{Configuration and numerical method}

The configuration chosen is that of the temporally developing mixing layer described in Miller \& Bellan (1999) and depicted in figure 1. For three-dimensional mixing layers, the streamwise $\left(x_{1}\right)$, cross-stream $\left(x_{2}\right)$ and spanwise $\left(x_{3}\right)$ coordinates are defined; for two-dimensional mixing layers only directions $x_{1}$ and $x_{2}$ exist. Periodic boundary conditions are used for the $x_{1}$ - and $x_{3}$-directions, and non-reflecting outflow conditions (Poinsot \& Lele 1992) are employed for the $x_{2}$-boundaries.

The appropriate initial conditions for simulating the evolution of mixing layers have been a topic of much discussion, especially for density stratified situations. According to stability theory (see Drazin \& Reid 1981), there are two issues that must be addressed: first, one must inquire about the basic (i.e. mean) flow, and then about the appropriate disturbance.

\subsection{The basic flow}

\subsubsection{Simulation conditions}

The present strategy is to choose a basic flow and further verify the validity of this choice. The initial cross-stream dependences of the mean velocity, temperature, and mass fraction profiles are specified using an error function profile, $\operatorname{erf}\left(\sqrt{\pi} x_{2} / \delta_{\omega, 0}\right)$, where the initial vorticity thickness is $\delta_{\omega, 0}$ and $\delta_{\omega}(t)=\Delta U_{0} /\left\langle\partial u_{1} / \partial x_{2}\right\rangle_{\max }$ with 


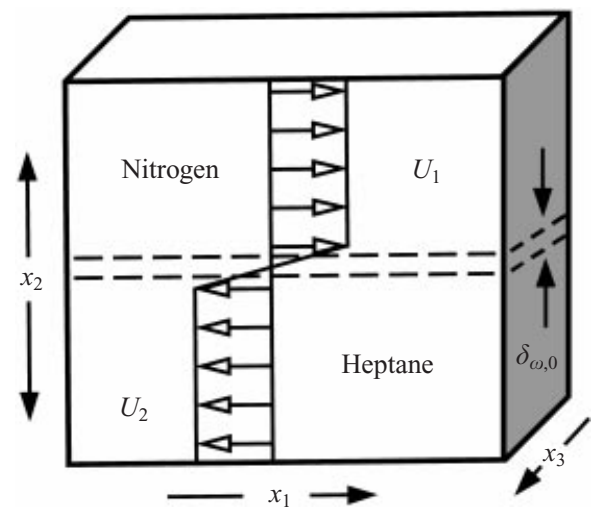

FIGURE 1. Physical configuration studied.

the brackets \langle\rangle indicating averaging over homogeneous $\left(x_{1}, x_{3}\right)$-planes for threedimensional layers, and over the $x_{1}$-direction for two-dimensional layers. The density profile is then calculated from the equation of state.

All simulations are conducted on a domain having $L_{1}=4 \lambda_{1}=29.16 \delta_{\omega, 0}$ where $\lambda_{1}$ is the smallest forcing wavelength in the $x_{1}$-direction (see below).

In order to obtain stationary vortices in the computational domain, we derived a relationship between the convective velocity, $U_{c}$, and the convective Mach number, $M_{c}$, by recalling that according to Papamoschou \& Roshko (1988), the perfect-gas spatial mixing layer convective velocity is

$$
U_{c}=\frac{U_{2} / c_{2}+\left(\sqrt{\gamma_{1} / \gamma_{2}}\right) U_{1} / c_{1}}{1 / c_{2}+\left(\sqrt{\gamma_{1} / \gamma_{2}}\right) / c_{1}}
$$

where the subscripts 1 and 2 denote the upper and lower stream, $\gamma$ is the perfect gas constant, and $c^{2}=\gamma R_{u} T / \mathrm{m}$. The condition of stationary vortices for a temporal mixing layer yields $U_{c}=0$, thereby providing a relationship between $U_{1}$ and $M_{c} \equiv$ $0.5\left(M_{c_{1}}+M_{c_{2}}\right)$ where $M_{c_{1}} \equiv\left(U_{1}-U_{c}\right) / c_{1}$ and $M_{c_{2}} \equiv\left(U_{c}-U_{2}\right) / c_{2}$ as follows:

$$
U_{1}=\frac{2 M_{c} c_{1}}{1+\sqrt{\gamma_{1} / \gamma_{2}}} \text { and } U_{2}=-U_{1} \frac{c_{2}}{c_{1}} \sqrt{\frac{\gamma_{1}}{\gamma_{2}}} \text {. }
$$

The above relations were derived under the assumption of calorically perfect gases, an assumption not met for the present conditions. Preliminary simulations conducted with a convective Mach number based on these relations resulted in translation of the vortices throughout the simulation, confirming that a correction is required for real fluids. For this purpose, a relatively simple correction is made by noting that $c_{1}$ and $c_{2}$ in (3.2) should be the true real-gas sound speeds $a_{s_{1}}$ and $a_{s_{2}}$, respectively, since they appear through the definition of the Mach number. However, the heat capacity ratios $\gamma_{1}$ and $\gamma_{2}$ result from applications of the calorically perfect relation $c^{2}=\gamma R_{u} T / m$. Therefore a simple correction is achieved by replacing the heat capacity ratio with an effective value, $\gamma^{*}$, which is related to the true sound speed through

$$
a_{s}^{2} \equiv \gamma^{*} R_{u} T / m=\gamma^{*} p /(\rho Z)
$$

where the compression factor $Z=p /\left(\rho T R_{u} / m\right)$ indicates the deviation from perfect 


\begin{tabular}{cccccc}
\hline Run & $T_{2}$ & $\rho_{2} / \rho_{1}$ & $N_{1} \times N_{2}\left(\times N_{3}\right)$ & $\alpha_{I K}$ & $\alpha_{B K}$ \\
1 & 600 & 12.88 & $200 \times 232$ & 0.1 & - \\
2 & 600 & 12.88 & $200 \times 232$ & 1.0 & - \\
3 & 600 & 12.88 & $200 \times 232$ & 5.0 & - \\
4 & 600 & 12.88 & $200 \times 232$ & - & 0.1 \\
5 & 600 & 12.88 & $200 \times 232$ & - & 1.0 \\
6 & 600 & 12.88 & $200 \times 232$ & - & 5.0 \\
7 & 900 & 4.27 & $200 \times 232$ & 0.1 & - \\
8 & 600 & 12.88 & $200 \times 232 \times 120$ & 0.1 &
\end{tabular}

TABLE 3. Simulation parameters. All runs have four initial vortices and two pairings: $M_{c, 0}=0.4$, $R e_{0}=400, T_{1}=1000 \mathrm{~K}$, and $p_{0}=60 \mathrm{~atm}$.

gas behaviour; one finds that

$$
\sqrt{\gamma_{1}^{*} / \gamma_{2}^{*}}=\left(a_{s_{1}} / a_{s_{2}}\right) \sqrt{\left(\rho_{1} Z_{1}\right) /\left(\rho_{2} Z_{2}\right)}
$$

since $p_{1}=p_{2}$. Using (3.2) and (3.4) one finds an expression valid for a general fluid

$$
U_{1}=2 M_{c} a_{s_{1}}\left[1+\left(\frac{a_{s_{1}}}{a_{s_{2}}}\right) \sqrt{\frac{\rho_{1} Z_{1}}{\rho_{2} Z_{2}}}\right]^{-1}, \quad U_{2}=-\sqrt{\frac{\rho_{1} Z_{1}}{\rho_{2} Z_{2}}} U_{1},
$$

where $\Delta U_{0}=U_{1}-U_{2}$ is the free-stream velocity difference across the layer. This derivation being only approximate, one cannot expect the vortices to stay truly stationary, a fact that can be observed for some of the simulations presented below.

Papamoschou \& Roshko (1988) discuss $M_{c}$ effects on the growth of spatially evolving, density-stratified mixing layers and show that for perfect gases the mixing layer growth is a function of $U_{2} / U_{1}, \rho_{2} / \rho_{1}$, the ratio of the perfect gas constants and $M_{c}$. Moreover, they show that the layer growth can be conveniently non-dimensionalized by that of the incompressible mixing layer (i.e. $M_{c}=0$ ) to obtain a consistent curve displaying an initial reduction and a further saturation with increasing $M_{c}$. In the present temporal study we do not address this compressibility effect, as all our simulations are conducted for the same value of $M_{c, 0}$ (see table 3). Here 'compressibility' is associated with the equation of state (i.e. $\kappa_{s}$ of (2.15)) and all other manifestations of compressibility (i.e. $\boldsymbol{\nabla} \cdot \boldsymbol{u}$ or local $M_{c}$ ) are interpreted in relation to this basic aspect of the fluid.

The reference viscosity is calculated from a specified value of the flow Reynolds number defined as

$$
R e_{0}=\frac{0.5\left(\rho_{1}+\rho_{2}\right) \Delta U_{0} \delta_{\omega, 0}}{\mu_{R}}
$$

where the free-stream densities are calculated from a specified constant initial flow pressure $\left(p_{0}\right)$, composition and free-stream temperature $\left(T_{1}\right.$ and $\left.T_{2}\right)$.

We note that according to (3.5), the density ratio affects the value of $\Delta U_{0}$ and the interpretation of the results should reflect this fact. This somewhat ambiguous situation is inherent in the nature of typical direct numerical simulations and is equivalent to that encountered when $R e_{0}$ is varied at constant $M_{c}: \mu_{R}$ changes since $\Delta U_{0}$ is constant, and this is despite the fixed value of the free-stream temperature (the free-stream temperature enters the calculation of $U_{1}$ through $M_{c}$ and thus through $a_{s_{1}}$ ), thereby introducing a similar ambiguity.

The conservation equations are solved numerically using the same numerical 
method as in Miller \& Bellan (1999): a fourth-order explicit Runge-Kutta temporal integration for all time derivatives and eighth-order-accurate central finite differences for all spatial derivatives. The simulations are performed on a HewlettPackard Exemplar parallel supercomputer using Message Passing Interface (MPI) subroutines for inter-processor communications and the equations are parallelized using three-dimensional domain decomposition.

\subsubsection{Confirmation of the validity of the basic flow profile}

Kozusko et al. (1996a) have investigated the structure of the basic flow for compressible mixing layers in binary mixtures considering the effect of variable properties, and have shown that the profiles are strongly dependent both on the species and on whether the lighter stream is faster or slower than the heavy stream. Kennedy \& Chen (1998) have performed a study of basic flow effects on the stability of planar jets under the assumptions of constant pressure, constant specific heats and unity $\mathrm{Pr}$, but they accounted for the effects of heat conduction and viscous dissipation. Since most of the focus of the present study is on the influence of the thermal diffusion factors for generally otherwise identical initial conditions equations, we explored the sensitivity of the one-dimensional (the direction chosen is that of the stratification, i.e. $x_{2}$ ) basic flow to the values of these factors. Four laminar calculations were thus performed with identical initial conditions but different values of $\alpha_{I K}$ and $\alpha_{B K}$ corresponding to simulations discussed in $\S 4$ (Runs $1,3,4$ and 6 of table 3 , respectively). These calculations were conducted using both 232 and 1024 grid points; the results are the same for both resolutions. The dynamic and thermodynamic variables are plotted in figure 2 at a non-dimensional time $t \Delta U_{0} / \delta_{\omega, 0}=100$, corresponding to the maximum simulation time examined for the perturbed layer (see $\S 4$ ). The illustrations show that the density and velocity laminar profiles are insensitive to the thermal diffusion factors at all times of the perturbed mixing layer simulations, and that as expected, the temperature and mass fraction show increased diffusion with increasing thermal diffusion factor. Thus, the one-dimensional results indicate that any effects of the basic flow profile relaxation on the density, velocity or momentum will be nearly identical in all of the mixing layer simulations - and therefore will not obfuscate the discussions of effects attributed to diffusion coefficients. We note that all four laminar calculations have the same initial density stratification. An additional question arises as to the appropriate basic flow profiles for comparing layers with a different stratification, given the fact that previous studies (e.g. Kozusko et al. 1996a) showed that the base state flow is affected by the composition. However, if basic flow profiles depending on the density ratio were specified, these would introduce a bias owing to their influence on the diffusion velocities which would then be altered. This simple argument highlights the difficulty in choosing totally uncontroversial basic flow profiles. For this reason, the same basic flow profiles will be used for all simulations presented in $\S 4$.

\subsection{Stability and forcing}

The stability of compressible mixing layers has been previously addressed by Maslow $\&$ Kelly (1971) who computed the stability curves for a stratified mixing layer between two streams (both spatial and temporal configurations), by Lu \& Lele (1994) who studied the effect of the density ratio on the growth of a spatial mixing layer, and by Kozusko et al. (1996b) who explored the influence of compressibility on the growth rate. Many additional interesting topics could be discussed in the context of stability of the compressible layers. These topics include the influence of the disturbance mode on the layer growth and the effect that it has on the domain size of the computation, 

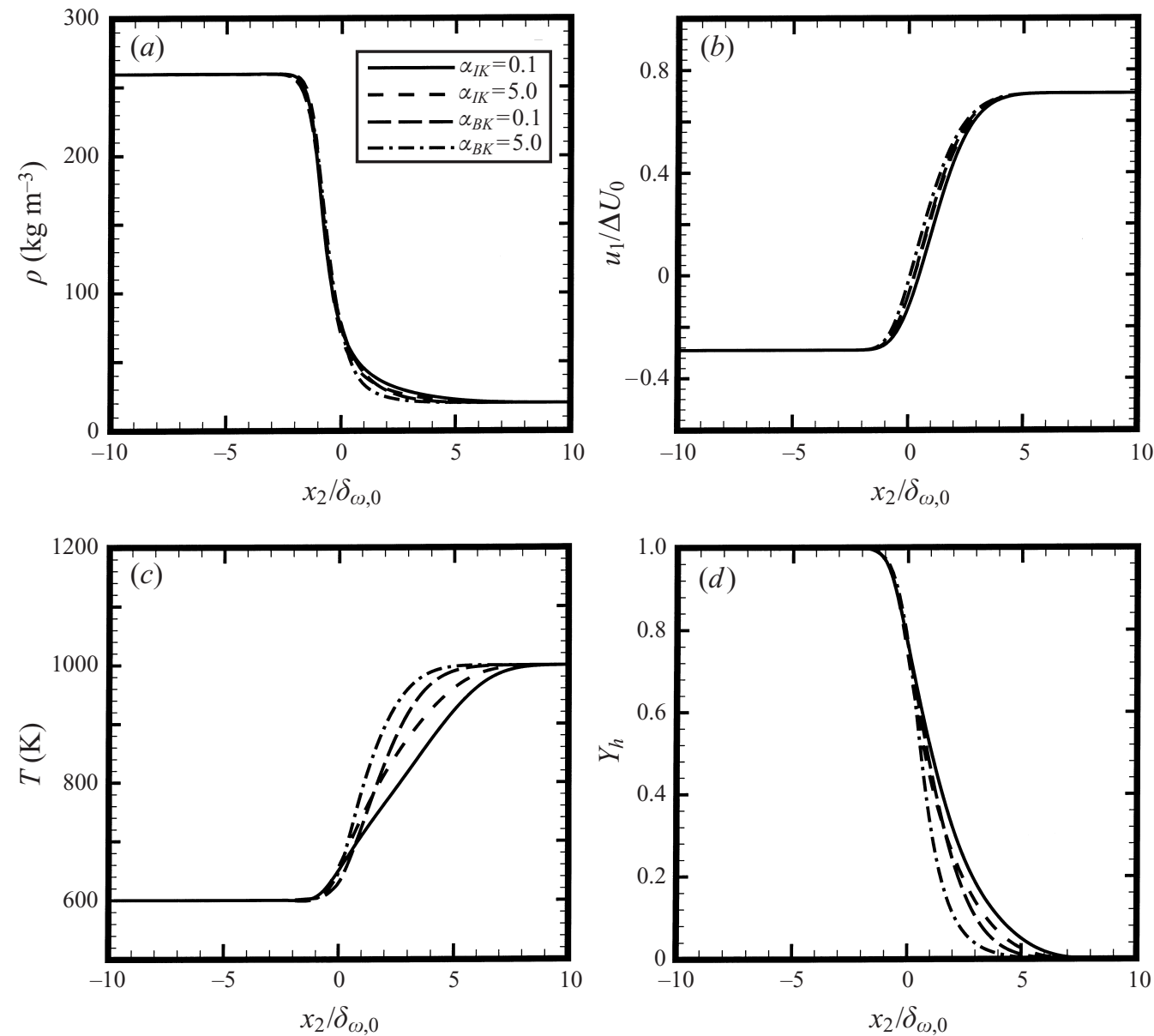

FiguRE 2. Basic flow profiles for a one-dimensional $\left(x_{2}\right)$ flow: density $(a)$, velocity $(b)$, temperature $(c)$ and mass fraction $(d)$ at the non-dimensional time $t \Delta U_{0} / \delta_{\omega, 0}=100$.

the influence of the Soret and Dufour diffusional processes on the most unstable wavelength, and the difference between exciting the layer using broadband versus large-amplitude discrete mode disturbances. All these topics are subjects of research in their own right, and are beyond the scope of this paper. We take here a more utilitarian view point and note that we are not interested in quantitatively matching our results with equivalent observations which, to our knowledge, do not exist. For this reason, we eliminate the possibility of using broadband disturbances since they are most commonly applied to three-dimensional mixing layers in order to achieve a more 'natural' turbulent flow; however, the present simulations are for laminar pre-transitional flows. Therefore, the use of exclusively small-amplitude broadband disturbances will only result in increasing the simulation time with little or no return on physical understanding. Also, since our emphasis is on the qualitative role of the thermal diffusion factors on the development of the layer rather than on growth, we do not seek a most unstable disturbance mode for exciting the layer. Instead, we choose a disturbance that is the most unstable incompressible mode (see Moser \& Rogers 1991), with a domain size equal to four times the most unstable wavelength (see below) and caution the reader about the possible influence of the domain size on the growth rate calculated at the end of each simulation. 
Specifically, initial forcing perturbations are added to the velocity field in order to excite in three-dimensions the growth of both spanwise and streamwise disturbances as in Miller \& Bellan (1999); for two-dimensional layers, only spanwise growth is excited. Sinusoidal velocity perturbations are superimposed on the base flow profile having wavelengths $L_{1}, L_{1} / 2$, and $L_{1} / 4$. All the simulations are performed by starting initially with four rollers and encompass two pairings; for all the simulations performed here $L_{1}=0.2 \mathrm{~m}$. The disturbances are first generated as a spanwise vorticity distribution of the form $\omega_{3}\left(x_{1}, x_{2}\right)=-\sum_{m=1}^{3} f\left(x_{2}\right)\left|A_{m} \sin \left(2 \pi x_{1} /\left(2^{m} \lambda_{1}\right)-\pi / 2\right)\right|$, where the cross-stream weighting is $f\left(x_{2}\right)=\exp \left(-\pi x_{2}^{2} / \delta_{\omega, 0}^{2}\right)$, and the relative amplitudes are $A_{1}=1, A_{2}=0.5$, and $A_{3}=0.35$. For three-dimensional simulations, an additional streamwise vorticity disturbance having wavelength $\lambda_{3}=\lambda_{1}$ is generated from the function $\omega_{1}\left(x_{2}, x_{3}\right)=$ $f\left(x_{2}\right)\left[\sin \left(2 \pi x_{3} / \lambda_{3}\right)+0.025 \sin \left(\pi x_{3} L_{3}\right)\right]$ (the second sinusoidal wave is used to break the symmetry between adjacent sets of streamwise vortices). The corresponding twodimensional or three-dimensional velocity disturbance is then calculated through the solution of the appropriate Poisson equation. The relative amplitudes of the specified spanwise $\left(F_{2 D}\right)$ and streamwise $\left(F_{3 D}\right)$ disturbances are equal to the ratio of the respective circulations relative to the approximated circulation of the mean velocity profile $\left(\lambda_{1} \Delta U_{0}\right)$.

\section{Results}

Displayed in table 3 are the parameters used in the simulations performed in this study; all simulations, except Run 7, use the same value of $\Delta U_{0}$. Although in the previous study of Harstad \& Bellan (2000) approximate values for the thermal diffusion factor were found, for a general binary species mixture such a result is not available. In the absence of this information, the natural tendency is to assume that the thermal diffusion factor is constant over the studied range of the state variables, and to assign it a value. Therefore, it is first important to identify the effect of the assumed constant value of the thermal diffusion factor. An additional complication arises in this argument since, as discussed above, one may define two thermal diffusion factors, only one of which converges to the kinetic theory value. Equation (2.21) shows that if one could measure one of these factors, the other one would be known as well since their difference is a thermodynamic function that can be calculated from the EOS. The question that comes forth addresses, then, the importance of the identity of the assumed constant thermal diffusion factor: Is there only a quantitative or is there also a qualitative difference between the predictions obtained on assigning constant values to the two forms of the thermal diffusion factor? Runs 1-3 and Runs 4-6 address the question of the value of each of the forms of the thermal diffusion factor, while comparisons between Runs 1 and 4,2 and 5 and 3 and 6 elucidate the effect of the identity of the assumed constant value thermal diffusion factor. Since these studies are qualitative by necessity and intent, Runs 1-6 represent two-dimensional simulations which are sufficient (in that a third dimension is not necessary) for the intended goal. The remaining Runs 7 and 8 are performed with a small value of $\alpha_{I K}$ to emulate the validated heptane-nitrogen model of Harstad \& Bellan (2000). While the initial heptane temperature for Runs 1-6 is approximately that of fuel jets injected in a diesel and gas turbine combustion chamber, Run 7 explores the effect of increasing the heptane initial temperature; we caution that the interpretation of Run 7 with respect to the other runs may be obfuscated by the different initial energy stratification which may affect the layer evolution. Finally, Run 8 is a threedimensional simulation, and its results are used to examine the possibility of the layer 
reaching a transitional state. All simulations extend to the end of the second pairing, reaching a final non-dimensional time that exceeds that of previous three-dimensional transitional gaseous and drop-laden shear layers of Miller \& Bellan (2000).

The presentation of the results focuses first on the global evolution of the mixing layer with the purpose of determining the effect of the thermal diffusion factors as discussed above. The influence of their value and identity on the final state of the layer is then illustrated by the instantaneous fields at the final simulation time. The discussion of the instantaneous fields is followed by the first-order statistics which aid in further assessments of the uncertainties associated with the thermal diffusion factors and also identify features of the layer truly unique to general fluids at high pressures. Finally, the second-order statistics confirm the conceptual picture developed from the zero- and first-order statistics and give an ultimate explanation for the particular aspects of the flow. Conclusions and further discussion are offered in the last section.

\subsection{Mixing layer evolution}

Hernan \& Jimenez (1982) have established through interpretation of their data that most of the growth of a mixing layer is due to entrainment, not to pairing, and that as much as $80 \%$ of the entrainment occurs during the lifetime of the individual spanwise vortex structures (the rollers) rather than during vortex pairing; this interpretation of their observations has been questioned by Cortesi et al. (1999) who point out that the definition of layer growth is not unique. The quantity that is most often used to measure such growth is the vorticity thickness, $\delta_{\omega}$, although sometimes it is preferable to measure it by the momentum thickness,

$$
\delta_{m}=\frac{1}{\left(\theta_{1}+\theta_{2}\right)^{2}} \int_{-L_{2} / 2}^{L_{2} / 2}\left(\theta_{2}-\left\langle\rho u_{1}\right\rangle\right)\left(\theta_{1}+\left\langle\rho u_{1}\right\rangle\right) \mathrm{d} x_{2}
$$

where $\theta_{1}=\left|\left\langle\rho u_{1}\right\rangle\right|_{x_{2}=L_{2} / 2}$ and $\theta_{2}=\left|\left\langle\rho u_{1}\right\rangle\right|_{x_{2}=-L_{2} / 2}$, because it is an integral quantity and therefore does not exhibit the fluctuations inherent in $\delta_{\omega}$. Cortesi et al. (1999) showed that several measures of entrainment, including the momentum thickness, are qualitatively similar. While the growth is primarily a consequence of entrainment, the product thickness defined as

$$
\delta_{p}=\iiint_{V} \rho Y_{p} \mathrm{~d} V
$$

in mass units, where $Y_{p}=2 \min \left(Y_{h}, Y_{n}\right)$, is a direct consequence of molecular mixing as explained by Cortesi et al. (1999). Both $\delta_{m} / \delta_{\omega, 0}$ and $\delta_{p} / \delta_{p, 0}$ are illustrated in figure 3 for Runs $1-8$ as a function of the non-dimensional time $t \Delta U_{0} / \delta_{\omega, 0}$.

As expected, all $\delta_{m} / \delta_{\omega, 0}$ curves (see figure $3 a$ ) coincide for two-dimensional layers up to the first rollup $\left(t \Delta U_{0} / \delta_{\omega, 0} \approx 25\right)$ after which differences occur: for example, all lower $T_{2}$ layers are thinner due to the larger heptane density (stronger density stratification) which impedes entrainment. The increased thickness of the larger $T_{2}$ layer is also attributable to the larger $\Delta U_{0}$ (353 versus $296 \mathrm{~m} \mathrm{~s}^{-1}$ ) which changes with initial stratification at fixed $M_{c}$, as noted above. For larger initial density stratification (see table 3), the layer has a larger inertia which hinders rollup and retards strong forcing effects (whose manifestations are the oscillations in the momentum thickness curves). In our context the inertia is characterized by $\rho u$, and since $\rho_{2} u_{2} / \rho_{1} u_{1}=\sqrt{Z_{1} \rho_{2} /\left(Z_{2} \rho_{1}\right)}>1$ (see table 3 ), this ratio is larger with increasing $\rho_{2} / \rho_{1}$ since $Z_{1} / Z_{2}=O(1)$. Indeed, if one defines a Reynolds number associated with each of the streams, $R e_{j} \equiv \rho_{j}\left|u_{j}\right|\left(L_{2} / 2\right) / \mu\left(T_{j}\right)$, it can be shown that $R e_{2} / R e_{1}=\left(\rho_{2} / \rho_{1}\right)^{1.2}\left(Z_{2} / Z_{1}\right)^{0.2}\left(m_{1} / m_{2}\right)^{0.7}$, and it is its magnitude that characterizes 

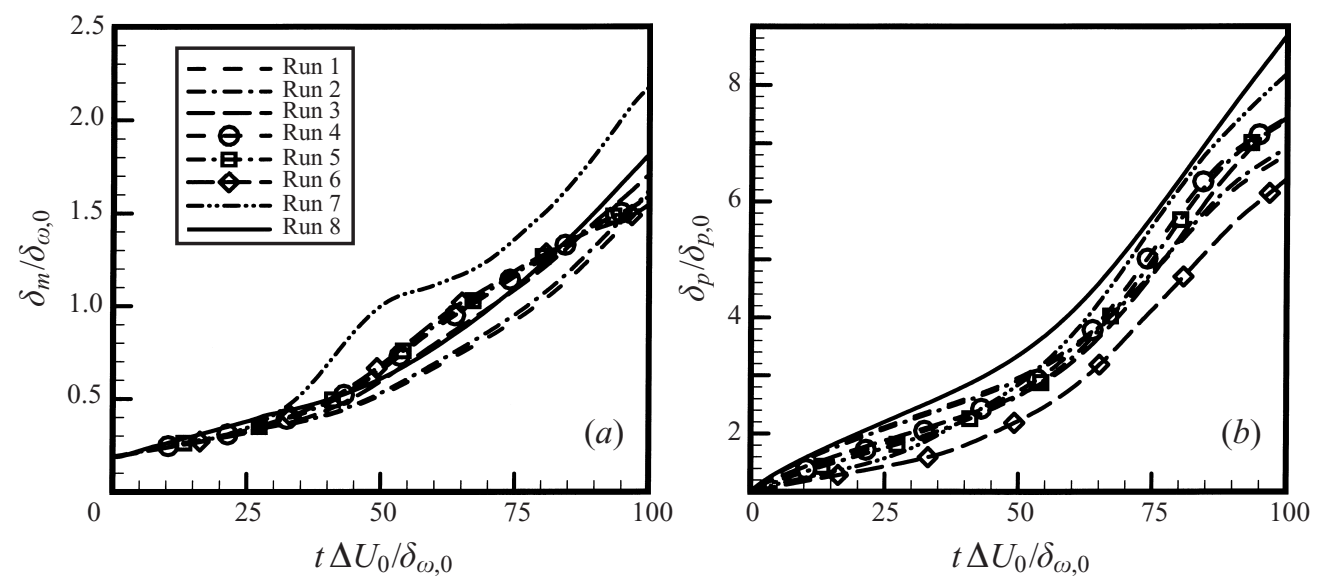

FIGURE 3. Non-dimensional momentum thickness $(a)$ and non-dimensional product thickness (b) evolution for the simulations listed in table 3.

the momentum-related initial strength of the density stratification; increasing density stratification is obtained with larger $R e_{2} / R e_{1}$ than unity. For example, the initial value of $R e_{2} / R e_{1}$ is 8.33 for Runs $1-6$ and Run 8, and it is 2.17 for Run 7, indicating the smaller density stratification in Run 7.

The layer growth (figure $3 a$ ) is quite insensitive to the value of the constant $\alpha_{B K}$, whereas increasing layer growth is obtained with increasing constant $\alpha_{I K}$ values (the reasons for this behaviour are explained below). One global indication of transition occurrence is a sudden and sharp increase of the momentum thickness and its sustained accelerated growth rate; however, transition to turbulence may occur only in three-dimensional layers (rather than two-dimensional layers) because of the necessary vortex stretching that is prerequisite to formation of turbulent small-scale structures. Flow visualizations (see below) reveal that transition does not occur in Run 8 despite the large simulation time and the completion of the two pairings; in contrast, for gaseous and drop-laden mixing layers, transition was obtained by Miller \& Bellan (2000) for similar $R e_{0}$ prior to $t \Delta U_{0} / \delta_{\omega, 0} \approx 100$. The inability to achieve turbulence transition in Run 8 is a direct consequence of the large density stratification which stabilizes the flow. The suppression/delay of transition may also be promoted by secondary effects (such as the appearance of strong density gradient regions) as will be discussed below.

A measure of the global mixing, rather than local mixing, is given by the product thickness plotted in figure 3(b); local mixing measures the effectiveness of the smallscale processes and the changes in the local distribution of the species, whereas global mixing encompasses the influence of all processes having an influence on the mass fraction distribution. The evolution of the product thickness can be explained by recalling that mixing can be limited by either small-scale processes or by the entrainment rate. Clearly, in examining the small scale processes, the largest mixing occurs in the three-dimensional layer because of the emerging turbulent small scales, as will be discussed below; in contrast, two-dimensional layer mixing is confined to molecular mixing which is weaker. In this sense, it can be stated that product thickness growth in two-dimensional layers is primarily due to stirring which stretches surfaces, not to mixing that relies on the small-scale diffusional processes. In three-dimensional layers, stirring may also be important as discussed by Atsavapranee \& Gharib (1997) 
in that once the small scales do appear, it promotes diffusional mixing. Prior to rollup, the weakest mixing (with the exception of the largest $\alpha_{B K}$ case) is that in the higher $T_{2}$ layer because of the reduced diffusivity. However, after pairing it is the Run 7 product thickness that becomes the largest due to the larger layer growth (figure $3 a$ ) resulting from the larger entrainment. With the exception of the largest constant- $\alpha_{B K}$ case which consistently exhibits the weakest mixing, all constant- $\alpha_{B K}$ simulations display a slightly reduced mixing up to pairing, and an enhanced mixing thereafter when compared to the constant- $\alpha_{I K}$ simulations; this effect is associated with the larger layer growth. For Run 6, the weak concentrational mixing is consistent with the larger molar flux cross-terms with increasing $\alpha_{B K}$, as discussed below and shown by the second-order statistics.

The identity of the assumed constant thermal diffusion factor has a direct influence on the response of the layer to forcing since by $t \Delta U_{0} / \delta_{\omega, 0} \approx 100$ (corresponding to the end of the second pairing) the constant- $\alpha_{I K}$ simulations, in contrast to the constant$\alpha_{B K}$ simulations, feature a sustained growth without the plateau characteristic of a strong forcing influence. What distinguishes the momentum evolution of the two types of layers is the evolution of the density stratification during rollup and pairing. With increased, fixed positive $\alpha_{I K}$, one imposes an impedance in the heat flux (see (2.17)) since $\nabla T$ and $\nabla Y_{h}$ are initially opposed, resulting in larger enthalpy gradients and larger density gradients (through the EOS). To explore the influence of the density stratification in vorticity production, we focused attention on Run 8 so as to include the effect of the stretching and tilting term which is absent in two-dimensional layers. The budget of the vorticity equation

$$
\frac{\mathrm{D} \boldsymbol{\omega}}{\mathrm{D} t}=(\omega \cdot \nabla) \boldsymbol{u}-(\nabla \cdot \boldsymbol{u}) \boldsymbol{\omega}-\nabla\left(\frac{1}{\rho}\right) \times \nabla p+\nabla \times\left(\frac{1}{\rho} \nabla \cdot \tau\right)
$$

where $\mathrm{D} / \mathrm{D} t$ is the substantial derivative and $\omega \equiv \nabla \times \boldsymbol{u}$, and of the vorticity magnitude equation

$$
\frac{\mathrm{D}\left(\omega^{2}\right)}{\mathrm{D} t}=2 \omega \cdot(\omega \cdot \nabla) \boldsymbol{u}-2(\nabla \cdot \boldsymbol{u}) \omega^{2}-2 \omega \cdot \nabla\left(\frac{1}{\rho}\right) \times \nabla p+2 \omega \cdot \nabla \times\left(\frac{1}{\rho} \nabla \cdot \tau\right)
$$

were both examined in contour plots of the spanwise vorticity and of the enstrophy in the between-the-braid plane, as well as $\left(x_{1}, x_{3}\right)$-plane averages. The first term in the above equations represents the stretching and tilting contribution, the second term describes the effect of dilatation, the third term is the baroclinic participation in vorticity production, and the last term portrays the viscous contribution. Contour plots (not shown) reveal that the dilatation term exhibits regions of both negative and positive contributions that may locally rival in magnitude the baroclinic term which is predominantly positive. Most of the activity of both terms is concentrated in regions of large density gradient magnitude (see figure 7) created during entrainment and mixing. Plane averages for the spanwise vorticity and the vorticity magnitude, illustrated in figures $4 a$ and $4 b$, respectively, show that on average the stretching term dominates all other terms, that the baroclinic and the viscous terms approximately balance each other in magnitude, and that the dilatation term is negligible.

When fixing $\alpha_{B K}$ positive, the molar flux cross-term impedance increases (see (2.18)) resulting in reduced mixing, and therefore in a smaller product thickness. However, fixing $\alpha_{B K}$ positive also yields very large $\alpha_{I K}$ (illustrated below in the discussion of the first-order statistics) as a result of the specific set of species and the chosen positive values for $\alpha_{I K}$ and $\alpha_{B K}$; the very large $\alpha_{I K}$ hinders the heat flux and helps maintain 

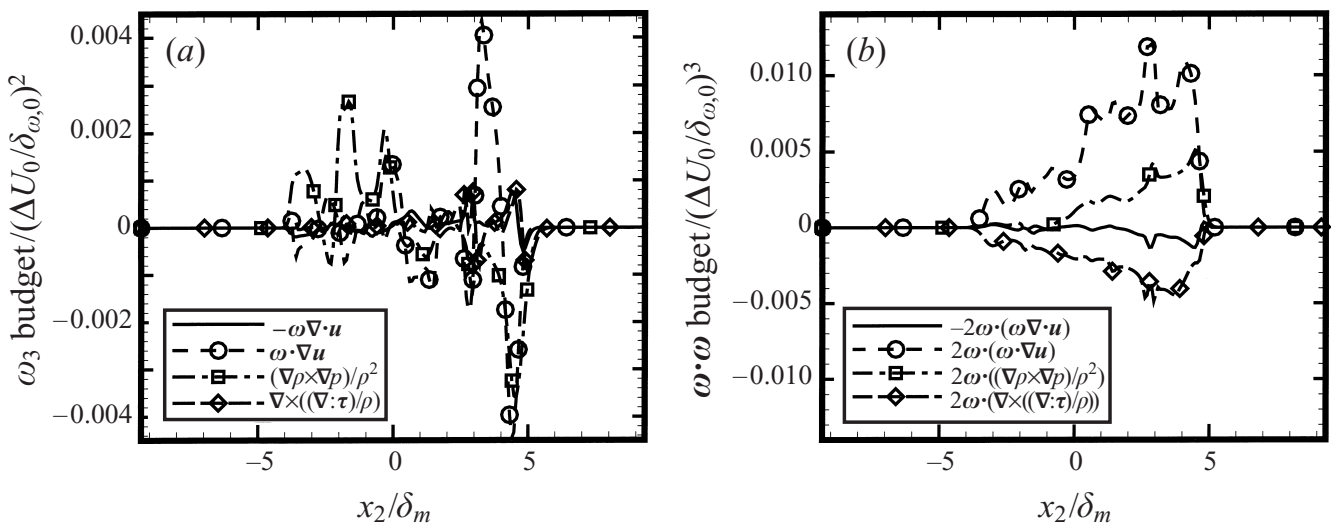

FiguRE 4. Plane-average contributions of different terms to the spanwise vorticity $(a)$ and the enstrophy $(b)$ for Run 8 . The calculations are for a between-the-braid plane at the non-dimensional time $t \Delta U_{0} / \delta_{\omega, 0}=100$.

large density gradients. Note though that fixing $\alpha_{B K}$ yields a larger value of $\alpha_{I K}$ (Runs 4-6) than fixing $\alpha_{I K}$ to have the same value as the fixed $\alpha_{B K}$ (Runs 1-3) due to the particular values of the thermodynamic term in (2.21). The larger values of $\alpha_{I K}$ indicate larger entrainment, explaining why the momentum thickness is larger for the fixed $\alpha_{B K}$ runs (except towards the end of the calculation). The larger role played by $\alpha_{I K}$ in determining entrainment can be understood from Lewis number considerations, as follows: Harstad \& Bellan (1999) showed that an effective Lewis number, $L e_{e f f}$, can be defined for supercritical conditions which is larger than the traditional Lewis number as it accounts for the effects of the difference in the molar enthalpy of the two species being transported through gradients. The large $L e_{\text {eff }}$ implies that the heat flux takes precedence over the mass flux in determining the state of the layer, particularly the density profile. A smaller $\alpha_{I K}$ implies less heat flux impedance, smaller enthalpy gradients and thus smaller density gradients, and therefore smaller vorticity production resulting in reduced entrainment when compared to the fixed $\alpha_{B K}$ case where the resulting $\alpha_{I K}$ is larger.

Therefore, it is apparent that $\alpha_{B K}$ influences mixing whereas $\alpha_{I K}$ influences entrainment; the fundamental reasons for these specific functions are discussed below when examining second-order statistics. This is consistent with $\alpha_{B K}$ determining the mass diffusion cross-term, and $\alpha_{I K}$ determining the heat flux cross-term and thereby changes in the density profile. Although changes in density profiles may be due to both changes in enthalpy and changes in mass fractions, it is expected that the changes in enthalpy will play a considerably larger role. This is consistent with the fact that changes in density due to mass fraction changes are expected to be moderate if the molecular weights of the species are similar, and is also consistent with the relatively larger effective thermal diffusivity.

\subsection{Instantaneous flow visualizations}

Flow visualizations can be very revealing of the instantaneous features of the layer which are removed when illustrating the cross-stream averages in two-dimensional or three-dimensional layers. Moreover, the instantaneous fields may provide one of the most direct ways to qualitatively compare characteristics of numerically simulated temporal mixing layers with those of experimentally observed spatially developing mixing layers. For this reason we present here contour plots of the primary variables 


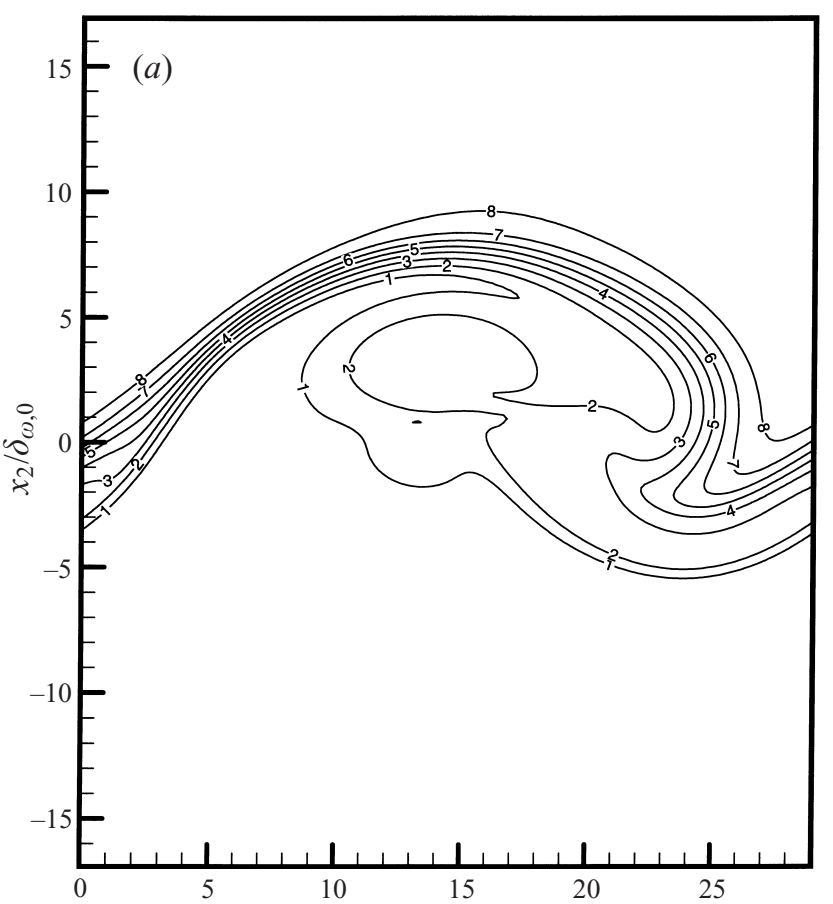

\begin{tabular}{|cc|}
\hline Level & $T$ \\
8 & 980 \\
7 & 930 \\
6 & 880 \\
5 & 830 \\
4 & 780 \\
3 & 730 \\
2 & 680 \\
1 & 630 \\
\hline
\end{tabular}

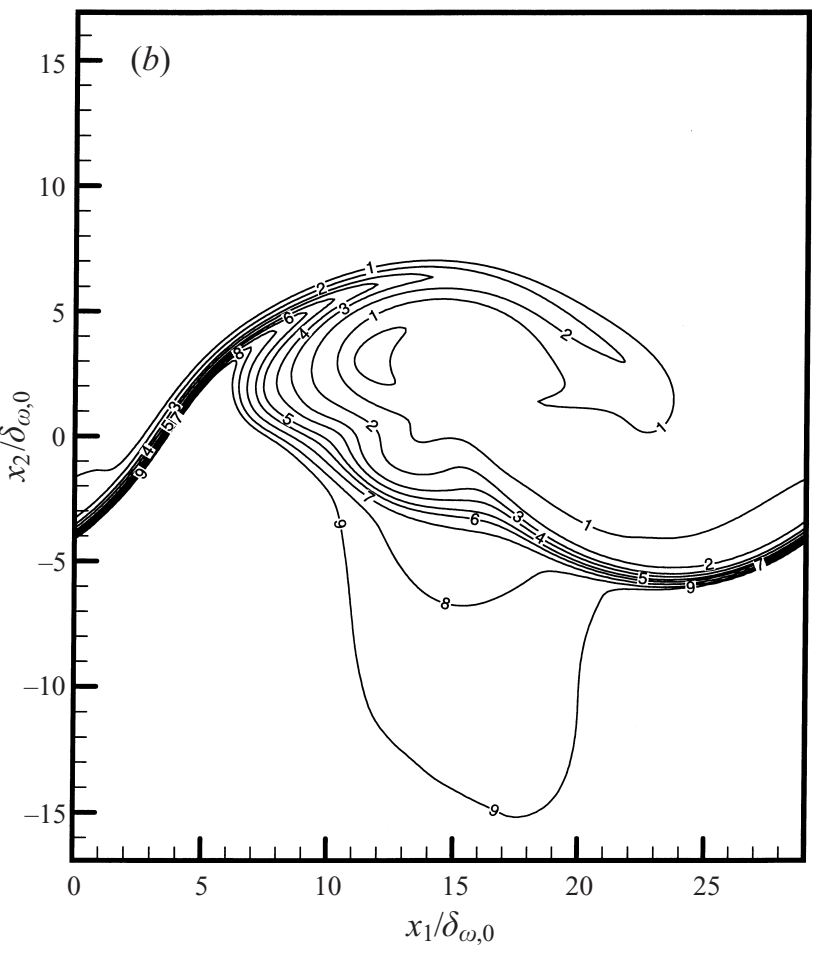

\begin{tabular}{|cc|}
\hline Level & $\rho$ \\
9 & 258 \\
8 & 248 \\
7 & 228 \\
6 & 198 \\
5 & 168 \\
4 & 128 \\
3 & 98 \\
2 & 68 \\
1 & 48 \\
\hline
\end{tabular}

FigURE 5. For caption see page 23. 


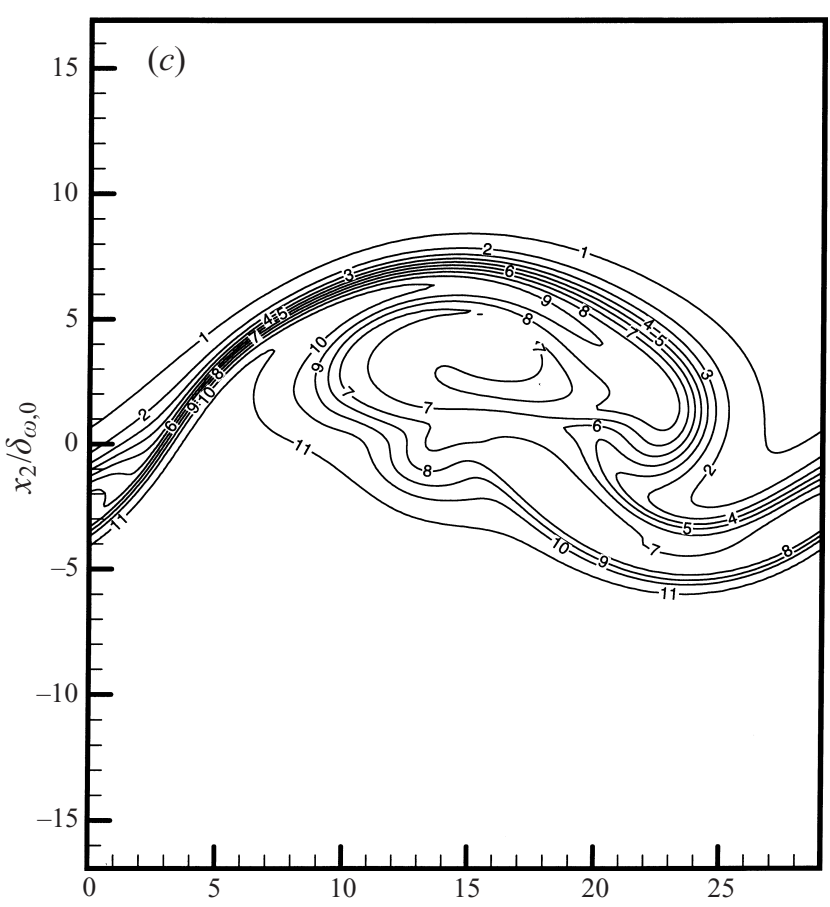

\begin{tabular}{|cl|}
\hline Level & $Y_{h}$ \\
11 & 0.99 \\
10 & 0.9 \\
9 & 0.8 \\
8 & 0.7 \\
7 & 0.6 \\
6 & 0.5 \\
5 & 0.4 \\
4 & 0.3 \\
3 & 0.2 \\
2 & 0.1 \\
1 & 0.01 \\
\hline
\end{tabular}

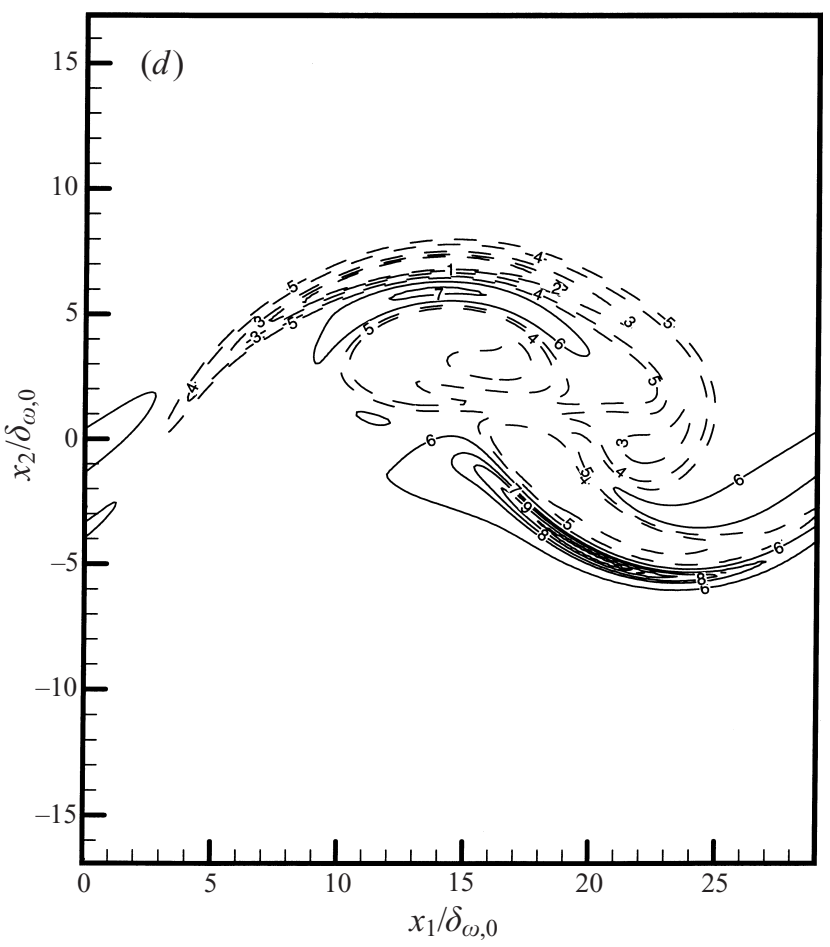

\begin{tabular}{|cc|}
\hline Level & $\omega_{3} /\left(\Delta U_{0} / \delta_{\omega, 0}\right)$ \\
10 & 0.505555 \\
9 & 0.455555 \\
8 & 0.355555 \\
7 & 0.255555 \\
6 & 0.045555 \\
5 & -0.155555 \\
4 & -0.255555 \\
3 & -0.455555 \\
2 & -0.555555 \\
1 & -0.615555 \\
\hline
\end{tabular}

FIGURE 5. For caption see page 23. 


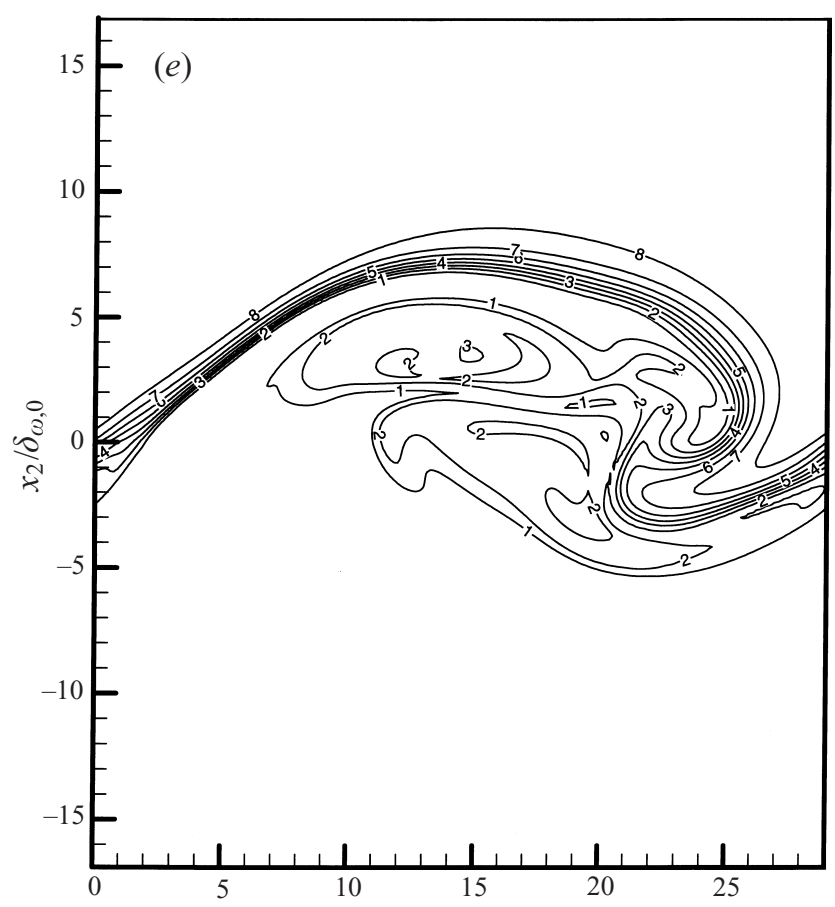

\begin{tabular}{|cc|}
\hline Level & $T$ \\
8 & 980 \\
7 & 930 \\
6 & 880 \\
5 & 830 \\
4 & 780 \\
3 & 730 \\
2 & 680 \\
1 & 630 \\
\hline
\end{tabular}

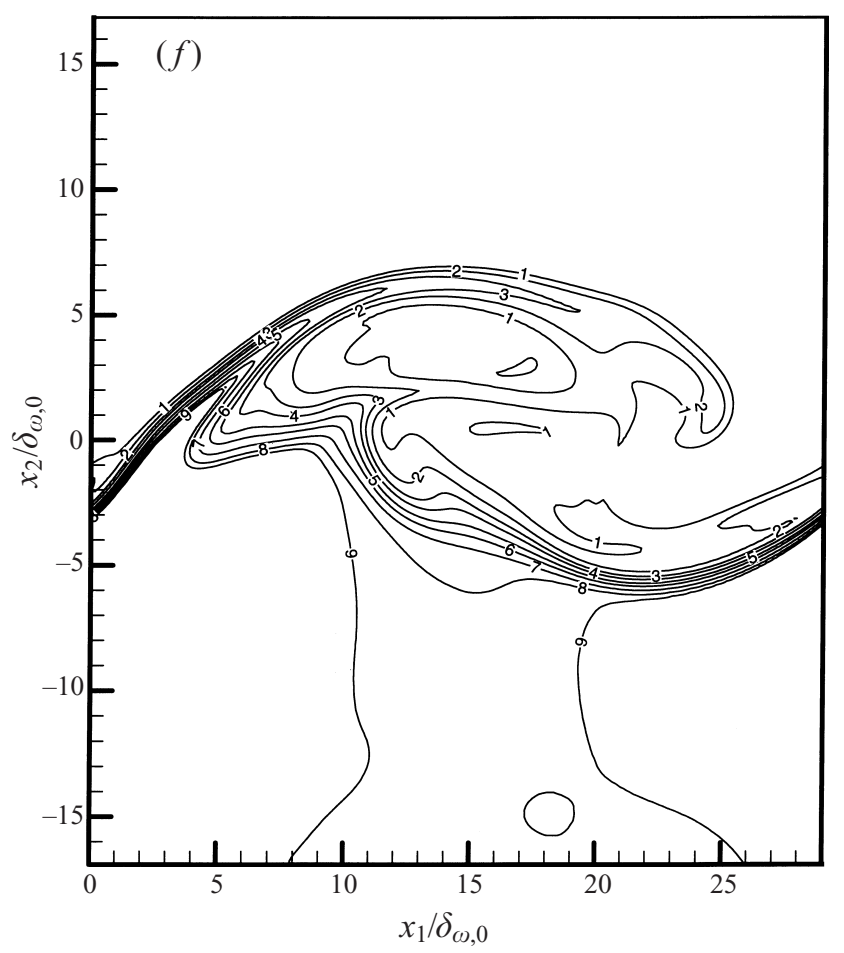

\begin{tabular}{|cc|}
\hline Level & $\rho$ \\
9 & 258 \\
8 & 248 \\
7 & 228 \\
6 & 198 \\
5 & 168 \\
4 & 128 \\
3 & 98 \\
2 & 68 \\
1 & 48 \\
\hline
\end{tabular}

FIGURE 5. For caption see facing page. 


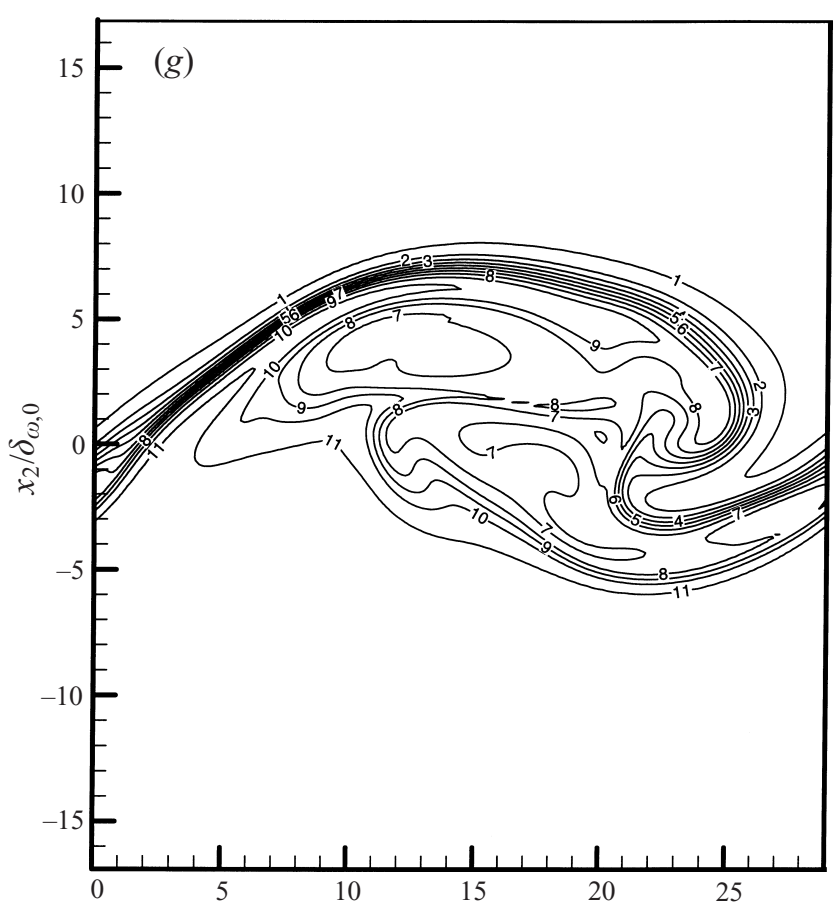

\begin{tabular}{|cl|}
\hline Level & $Y_{h}$ \\
11 & 0.99 \\
10 & 0.9 \\
9 & 0.8 \\
8 & 0.7 \\
7 & 0.6 \\
6 & 0.5 \\
5 & 0.4 \\
4 & 0.3 \\
3 & 0.2 \\
2 & 0.1 \\
1 & 0.01 \\
\hline
\end{tabular}

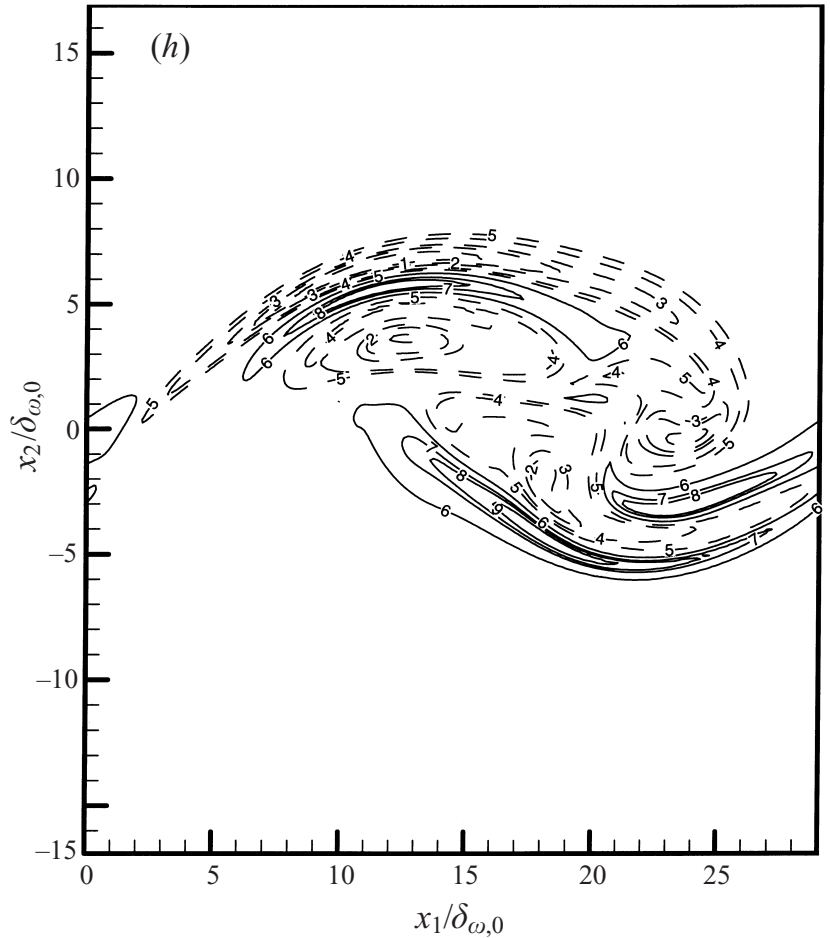

\begin{tabular}{|cc|}
\hline Level & $\omega_{3} /\left(\Delta U_{0} / \delta_{\omega, 0}\right)$ \\
9 & 0.505555 \\
8 & 0.355555 \\
7 & 0.255555 \\
6 & 0.045555 \\
5 & -0.155555 \\
4 & -0.255555 \\
3 & -0.455555 \\
2 & -0.555555 \\
1 & -0.7155555 \\
\hline
\end{tabular}

FiguRE 5. Instantaneous temperature in degrees Kelvin $(a, e)$, density in $\mathrm{kg} \mathrm{m}^{-3}(b, f)$, heptane mass fraction $(c, g)$ and non-dimensionalized spanwise vorticity $(d, h)$ for Run $1(a-d)$ and Run $4(e-h)$ at the non-dimensional time $t \Delta U_{0} / \delta_{\omega, 0}=100$. 
and of the quantities that are most distinctive to supercritical fluid flows at the final simulation time $t \Delta U_{0} / \delta_{\omega, 0}=100$.

\subsubsection{Two-dimensional layers}

Since the purpose of the two-dimensional simulations is studying the influence of the identity and value of the assumed constant thermal diffusion factors and that of the initial temperature of the hydrocarbon stream, we present here only those results that are pertinent to addressing this issue; we defer the more complete discussion of the flow characteristics to the section on the three-dimensional flow visualizations.

In figure 5 we illustrate the temperature ( $a$ and $e$ ), density $(b$ and $f)$ and heptane mass fraction profiles ( $c$ and $g$ ), as well as the non-dimensionalized spanwise vorticity $(d$ and $h$ ) for Run $1(a-d)$ and Run $4(e-h)$. Comparison of the primary-variable profiles shows both a larger magnitude of the temperature gradient due to the large positive $\alpha_{I K}$, and considerably more structure in Run 4, implying more developed smallscale structure and more fluctuations (see discussion on second-order statistics) for the assumed constant $\alpha_{B K}$ simulation. This observation is confirmed by comparisons of the spanwise vorticity in figures $5(d)$ and $5(h)$ where the contour levels were intentionally chosen the same, but the highest level appearing in figure $5(h)$ does not appear in figure $5(d)$. We discuss the fundamental reasons for these aspects in the statistical analysis below.

The spanwise vorticity is often used to visualize the large-scale rollers in low- $M_{c}$ mixing layers because vorticity with closed isosurfaces is a reasonable indicator of vortices. In this study, owing to the initial mean velocity profile, the corresponding initial spanwise vorticity is negative everywhere in the layer. Since the only two mechanisms for increasing vorticity in two-dimensional simulations are the compressibility through $\nabla \cdot \boldsymbol{u}$ and the density gradients, the appearance of positive vorticity over a wider region in the layer in Run 4 implies higher compressibility and/or stronger density gradients effects. As discussed above, density gradients are stronger in Run 4 than in Run 1, and examination of Mach number contours (not shown) indeed leads to the conclusion that Run 4 exhibits higher local compressibility than Run 1; and figure 14 below confirms this interpretation of the results as well.

To explore the effect of the value of the thermal diffusion factors we examined visualizations (not shown) of the non-dimensionalized spanwise vorticity for Runs 3 and 6 and compared them with those of Runs 1 and 4 . Increasing the value of the thermal diffusion factor, or changing the identity of the constant thermal diffusion factor from IK to BK, induces increasing distortion in these features of the layer.

\subsubsection{Three-dimensional layers}

\section{Primary variables}

Portrayed in figure 6 are the temperature $(6 a)$, density $(6 b)$ and heptane mass fraction $(6 c)$ in the braid for Run 8 ; the contour plots for the between-the-braid plane are similar. As the layer rolls up, fluid from the cooler, heptane region is entrained and mixes with the hotter, nitrogen fluid producing a highly non-uniform distribution of heptane in the roller; had the layer developed to transition (see discussion below), the heptane distribution would have been more uniform across the layer. The large density stratification of the layer $\left(\rho_{2} / \rho_{1}=12.88\right.$; see table 3$)$ is responsible for the relatively weak roller that results from the pairing of the four initial rollers.

In figure 7 we display the density gradient magnitude in the braid $(7 a)$ and in between-the-braid ( $7 b$ ) planes. The most prominent feature in figure 7 is the existence of spatially concentrated regions of strong density variation. This distinctive feature is 
Direct numerical simulations of supercritical heptane-nitrogen mixing layers

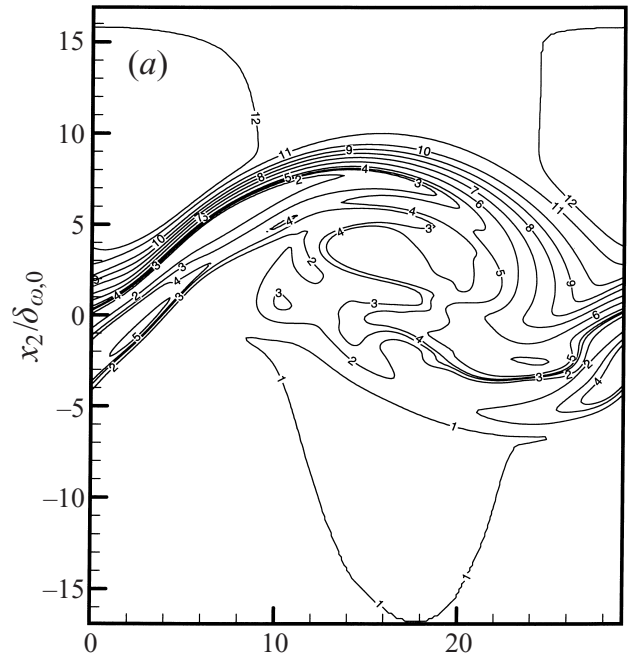

\begin{tabular}{|cc|}
\hline Level & $T$ \\
12 & 1000 \\
11 & 980 \\
10 & 930 \\
9 & 880 \\
8 & 830 \\
7 & 780 \\
6 & 730 \\
5 & 680 \\
4 & 660 \\
3 & 650 \\
2 & 630 \\
1 & 600 \\
\hline
\end{tabular}

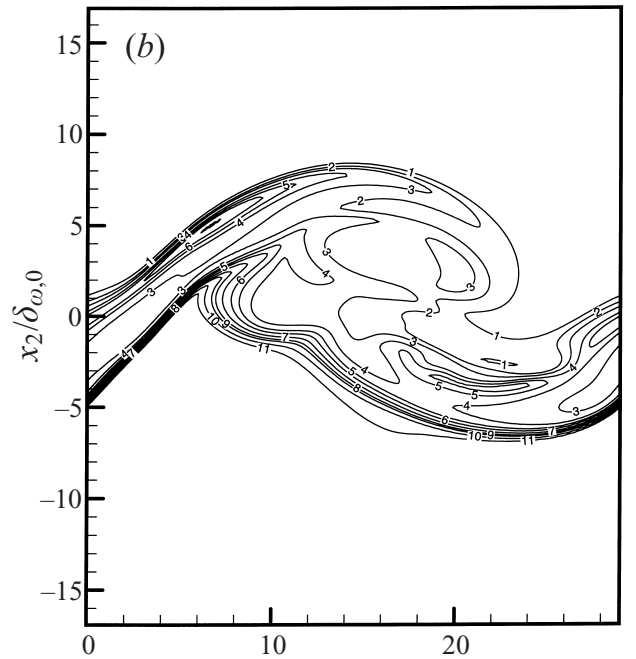

\begin{tabular}{|cc|}
\hline Level & $\rho$ \\
11 & 250 \\
10 & 230 \\
9 & 200 \\
8 & 180 \\
7 & 150 \\
6 & 120 \\
5 & 100 \\
4 & 80 \\
3 & 60 \\
2 & 50 \\
1 & 40 \\
\hline
\end{tabular}

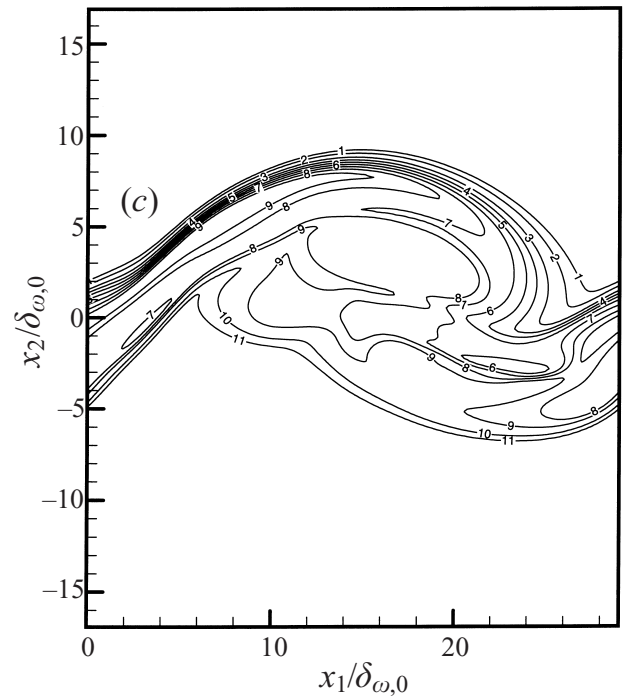

\begin{tabular}{|cl|}
\hline Level & $Y_{h}$ \\
11 & 0.99 \\
10 & 0.95 \\
9 & 0.8 \\
8 & 0.7 \\
7 & 0.6 \\
6 & 0.5 \\
5 & 0.4 \\
4 & 0.3 \\
3 & 0.2 \\
2 & 0.1 \\
1 & 0.05 \\
\hline
\end{tabular}

FIGURE 6. Temperature in degrees Kelvin $(a)$, density in $\mathrm{kg} \mathrm{m}^{-3}(b)$ and heptane mass fraction $(c)$ in the braid plane for Run 8 at the non-dimensional time $t \Delta U_{0} / \delta_{\omega, 0}=100$ 


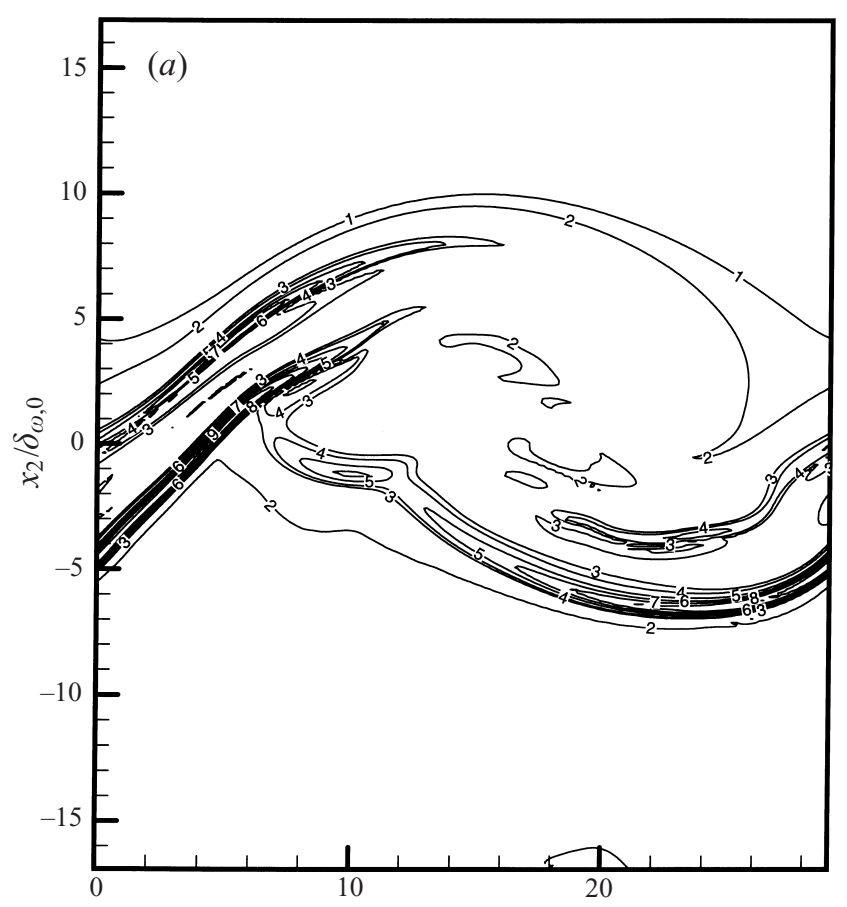

\begin{tabular}{|cc|}
\hline Level & $|\nabla \rho|$ \\
10 & 94725 \\
9 & 75000 \\
8 & 55000 \\
7 & 45000 \\
6 & 35000 \\
5 & 25000 \\
4 & 15000 \\
3 & 10500 \\
2 & 500 \\
1 & 50 \\
\hline
\end{tabular}

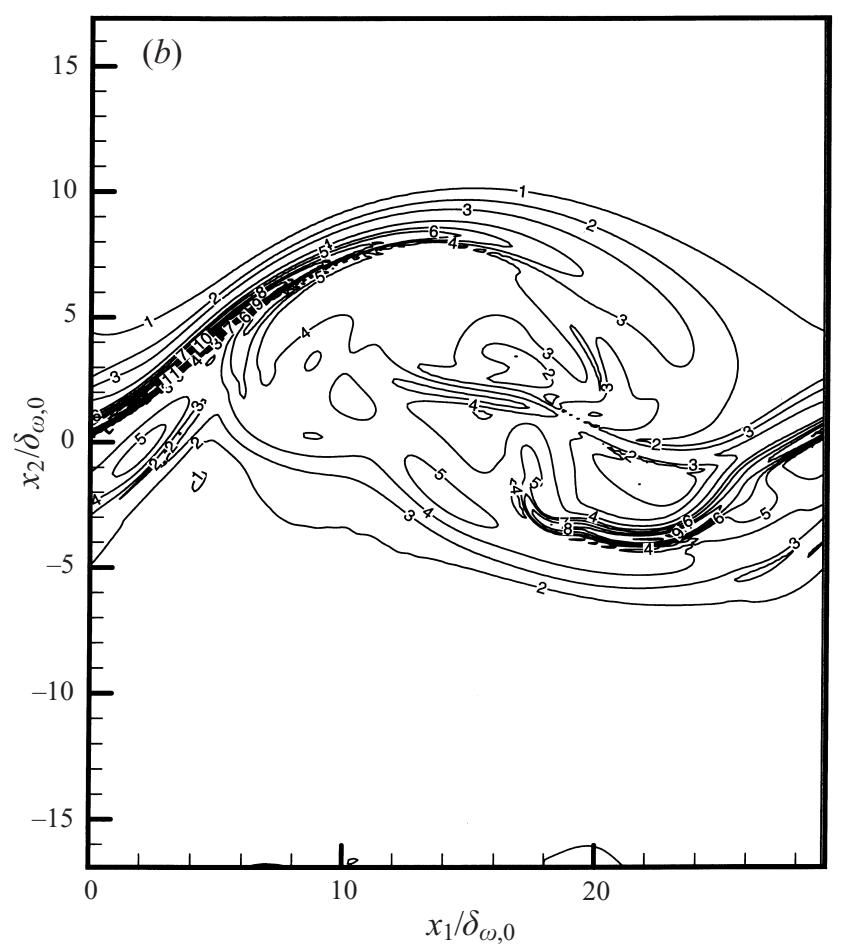

\begin{tabular}{|cc|}
\hline Level & $|\boldsymbol{\nabla} \rho|$ \\
12 & 85000 \\
11 & 75000 \\
10 & 65000 \\
9 & 50000 \\
8 & 35000 \\
7 & 25000 \\
6 & 15000 \\
5 & 10000 \\
4 & 5000 \\
3 & 1500 \\
2 & 500 \\
1 & 50 \\
\hline
\end{tabular}

FIGURE 7. Density gradient magnitude (in $\mathrm{kg} \mathrm{m}^{-4}$ ) in the braid $(a)$ and in between the braid $(b)$ planes for Run 8 at the non-dimensional time $t \Delta U_{0} / \delta_{\omega, 0}=100$. 


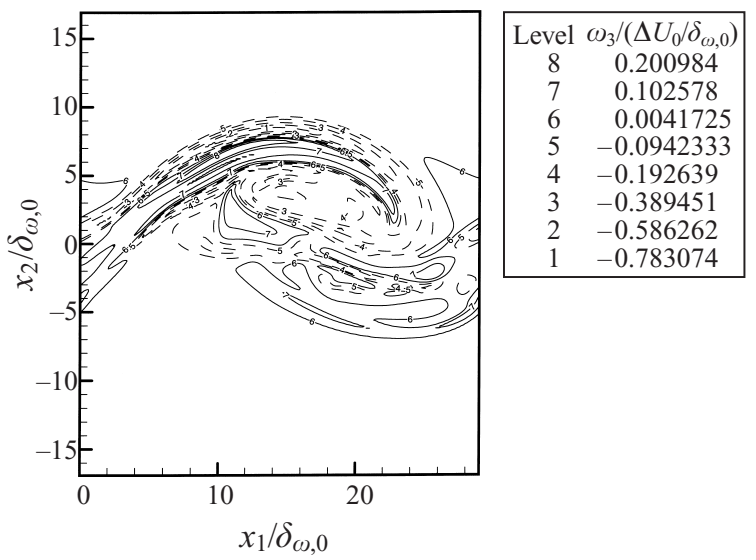

FIGURE 8 . Spanwise vorticity in the braid plane for Run 8 at the non-dimensional time $t \Delta U_{0} / \delta_{\omega, 0}=100$.

not exhibited by density-unstratified temporal mixing layers and, as will be discussed below, plays a crucial role in the understanding of the evolving turbulent scales in the flow.

\section{Turbulent scales development}

In contrast to two-dimensional mixing layers, in three-dimensional mixing layers there are four mechanisms for affecting vorticity (see (4.1)): dilatation, viscous dissipation, density gradient effects and vortex stretching; therefore, the increased occurrence of positive spanwise vorticity with respect to the two-dimensional layers may be a direct indication of vortex stretching, creation of small scales and transitions to turbulence. Figure 8 illustrates the spanwise vorticity in the braid; the results are similar for the between-the-braid plane. Clearly, very little positive vorticity has been generated, a fact that will be documented below in the timewise first-order statistics. This confirms that mixing is strongly entrainment limited over the entire simulation time, as also indicated by the evolution of the momentum and product thickness.

The delayed and sometimes suppressed development of turbulent scales in supercritical mixing layers is due to both the initial density stratification and to the formation of the sharp density gradient regions as illustrated in figures $7(a)$ and $7(b)$. For example, Hannoun, Fernando \& List (1988) observed that a density interface is very effective at damping turbulent eddies, being qualitatively similar to a rigid flat plate. Large eddies impinging on a density interface were found to 'bounce' back without significant entrainment of unstirred fluid instead of overturning. The same conclusion was reached by Briggs et al. (1998) who examined shear-free stably stratified mixing layers under the Boussinesq (i.e. constant density) approximation. Since the layer growth depends primarily on entrainment, and since it is this accelerated growth that promotes the appearance and evolution of the small turbulent scales, this damping mechanism could be enhancing the effect of the initial density stratification. The coupling between entrainment and mixing is manifested by the fact that the vertical position of any portion of fluid depends upon its density which is the result of mixing. In fact, Briggs et al. (1998) note that without the large-scale overturns, transfer of energy from the large to the small scale diminishes, thereby hindering transition to turbulence. To ascertain the effect of the large- $|\nabla \rho|$ regions in influencing the damping of the emerging small scales, Okong'o \& Bellan (2000) examined the dissipation using an enlarged database corresponding to Run 8 . The results showed that at the 
time following the culminating global positive spanwise vorticity, most of the activity was concentrated at locations of large $|\nabla \rho|$. This dissipation mechanism was considered responsible for the lack of transition, as formed small scales were damped by the region of large $|\nabla \rho|$ which acted similarly to a material interface. The behaviour of the layer was shown to be the direct consequence of the two competing processes of entrainment producing strong density gradients (a stabilizing effect) and mixing reducing the density gradients (a destabilizing effect).

\section{Thermodynamic variables}

The compression factor, $Z$, and the mass diffusion factor, $\alpha_{D}$, are measures of departures from perfect gas and ideal mixture behaviour, respectively; for a perfect gas $Z=1$ and for an ideal mixture $\alpha_{D}=1$, these being the situations that are generally investigated. Detailed discussion about real gases can be found in Prausnitz et al. (1986), and Cussler (1984) comments upon the value of the mass diffusion factor in different thermodynamic regimes.

Figure 9 shows that the layer displays considerable departures from the typical perfect gas, ideal mixture situation. Both in the braid plane (figure $9 a$ ) and in the between-the-braid plane (not shown) the largest departures in $Z$ occur in the heptane region as well as in the highest density gradient regions identified in figure $7 a$ and $7 b$. In contrast to $Z$, most of the departure from mixture ideality (see figure $9 b ; \alpha_{D}$ has the same qualitative behaviour in the between-the-braid plane) occurs, as expected, in the mixed fluid, and also, similarly to $Z$ departures from unity, in the high density gradient regions. Since the largest $\alpha_{D}$ departures from unity are in the highest density gradient regions, this means that the observed wisps (which are in the highest density gradient regions) enclose a fluid that is molecularly mixed $\left(\alpha_{D}<1\right)$ but that will tend to resist further mixing because of non-ideality (the Fickian diffusive term tends to weaken as it progresses because it decreases $\alpha_{D} D$ as $\alpha_{D}$ decreases). Therefore we have identified at supercritical conditions a suppressed diffusional (molecular) mixing mechanism that is a direct consequence of thermodynamics through mixture non-ideality (i.e. $\alpha_{D}$ ) and effectively decreases the mass diffusivity (see (2.19)).

The general conclusions from the visualizations are that the choice of constant $\alpha_{B K}$, with consequent large $\alpha_{I K}$ and reduced heat flux, promotes the maintenance of strong density gradients, shear and vorticity as especially illustrated by the results of Run 6 . In contrast, constant- $\alpha_{I K}$ simulations promote diffusional concentration mixing, this being a direct consequence of a large enthalpy difference $((2.21))$, producing a large magnitude, negative $\alpha_{B K}$ and strong coupling in the mass flux. Therefore, constant moderate $\alpha_{I K}$ combined with $\alpha_{D}$ effects are contrary in that the former enhances the molar fluxes $\left(\alpha_{B K}<0\right)$ and smears mass fraction gradients inducing a greater diffusional mixing. In contrast, both $\alpha_{D}$ and constant $\alpha_{B K}$ inhibit molar fluxes; the resulting large $\alpha_{I K}$ from the corresponding value of $\alpha_{B K}$ inhibits the heat flux. Since the relationship between $\alpha_{I K}$ and $\alpha_{B K}$ is determined by thermodynamics, it is thus species-pair dependent, and therefore these conclusions regarding $\alpha_{I K}$ and $\alpha_{B K}$ are not universal.

\subsection{Statistical profiles}

The flow visualization at the final time indicated that there are important differences between the detailed results of constant $-\alpha_{I K}$ and constant- $\alpha_{B K}$ simulations; however, it is only through statistical analysis that the fundamental reasons for these differences can be identified. Therefore, we devote this section to calculations of mean values and fluctuating statistical quantities at the final time in order to isolate the influence of the assumed constant thermal diffusion factors upon both the other transport properties 


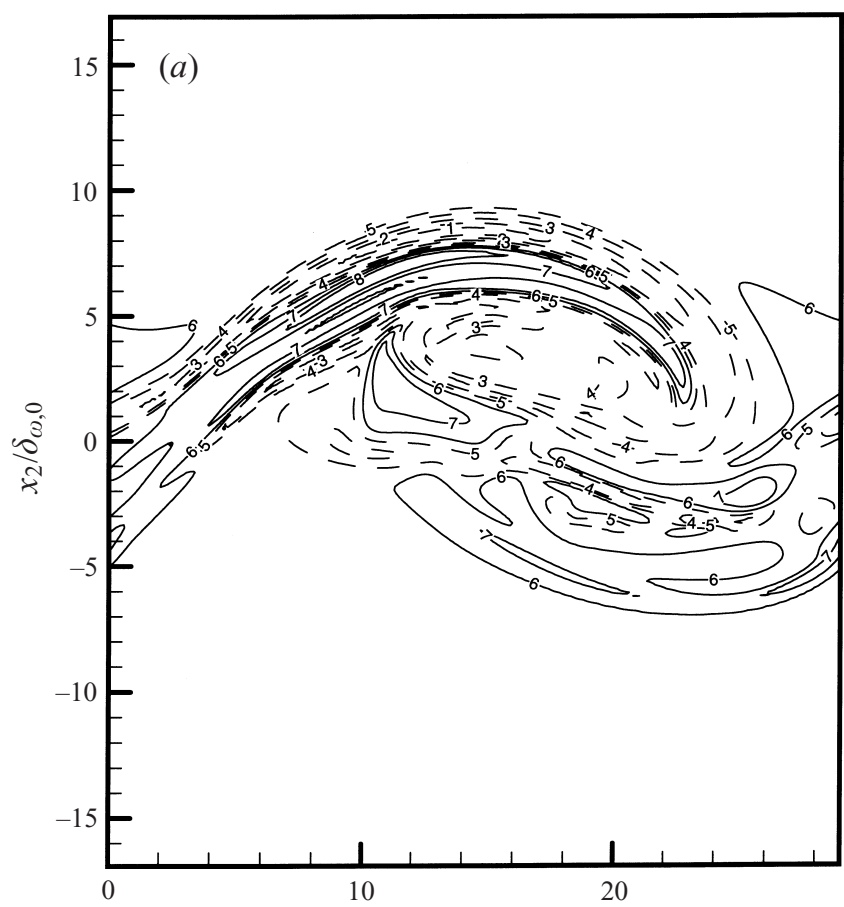

\begin{tabular}{|cl|}
\hline Level & \multicolumn{1}{c|}{$Z$} \\
11 & 0.99 \\
10 & 0.95 \\
9 & 0.9 \\
8 & 0.85 \\
7 & 0.8 \\
6 & 0.75 \\
5 & 0.7 \\
4 & 0.65 \\
3 & 0.6 \\
2 & 0.55 \\
1 & 0.5 \\
\hline
\end{tabular}

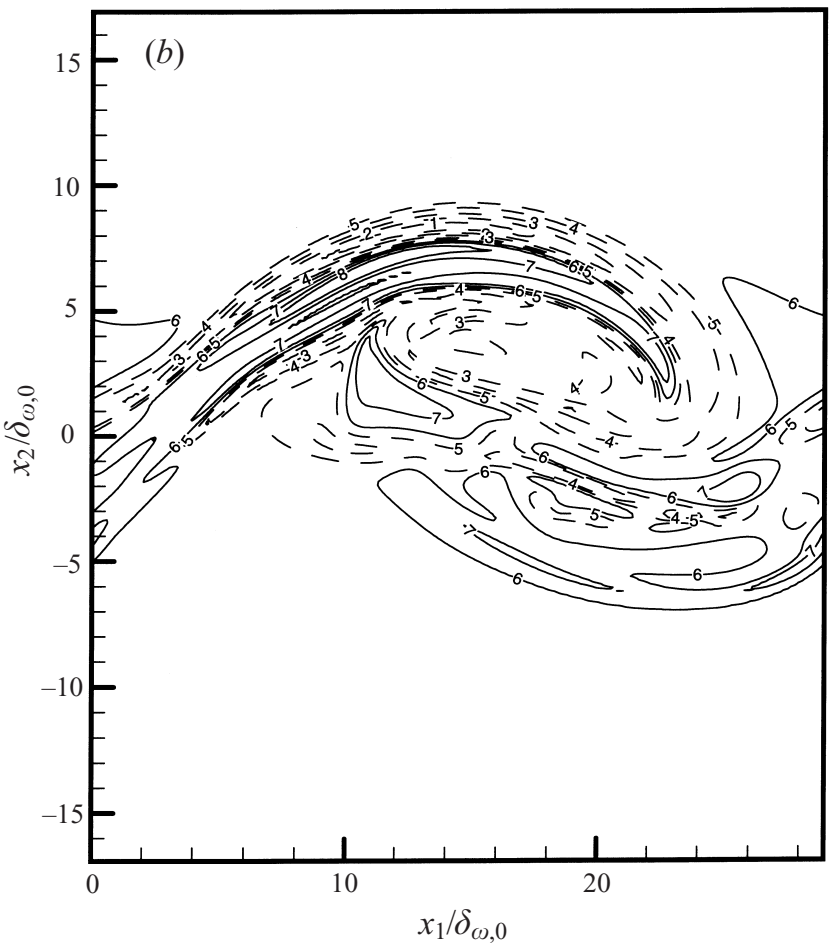

\begin{tabular}{|cc|}
\hline Level & $\alpha_{D}$ \\
7 & 0.97 \\
6 & 0.92 \\
5 & 0.87 \\
4 & 0.82 \\
3 & 0.77 \\
2 & 0.72 \\
1 & 0.67 \\
\hline
\end{tabular}

FIGURE 9. The compression factor $(a)$ and the mass diffusion factor $(b)$ in the braid plane at the non-dimensional time $t \Delta U_{0} / \delta_{\omega, 0}=100$. 

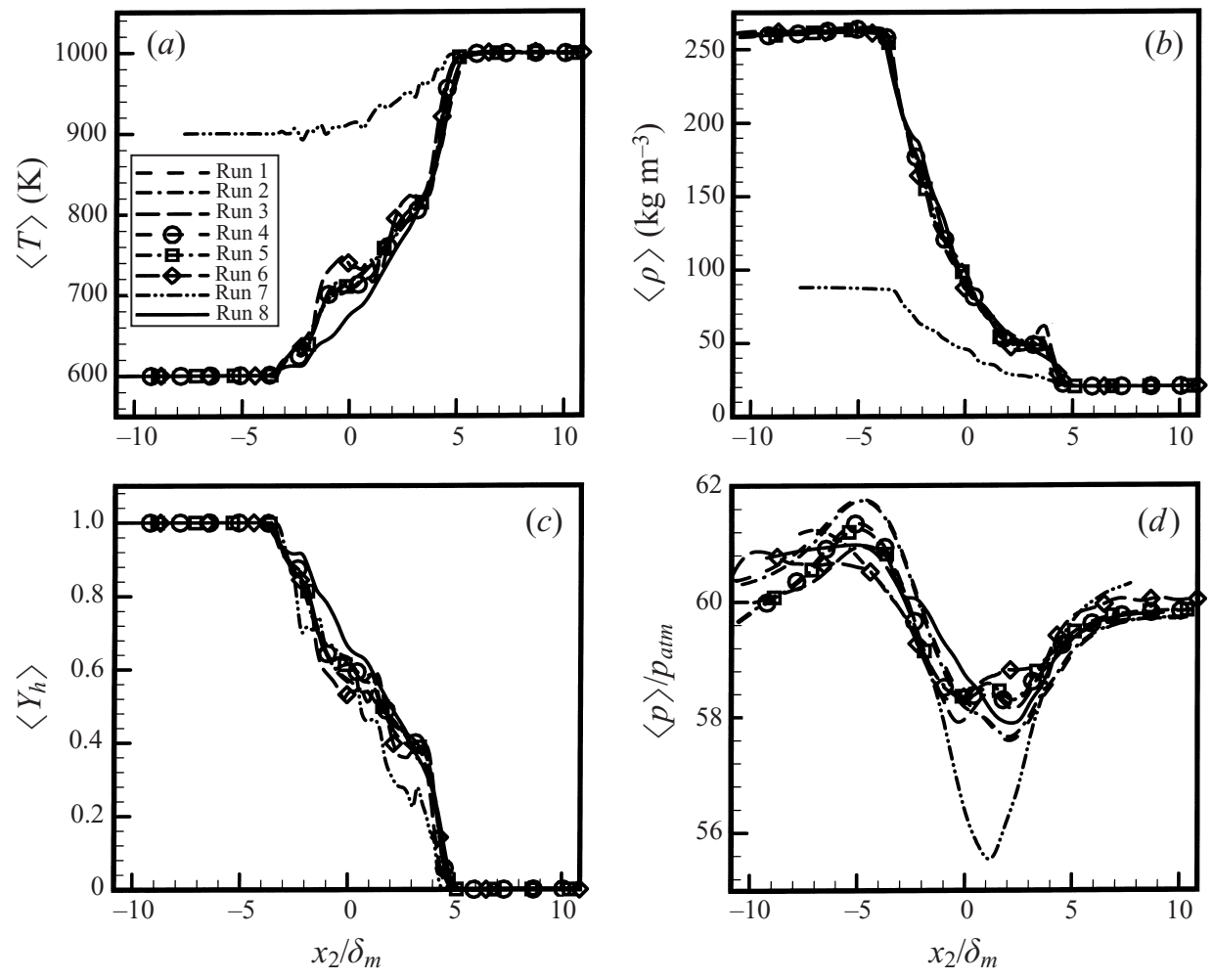

FIGURE 10. Mean primary variables as a function of a non-dimensional cross-stream variable: temperature $(a)$, density $(b)$, heptane mass fraction $(c)$ and pressure $(d)$ for Runs $1-8$ at the non-dimensional time $t \Delta U_{0} / \delta_{\omega, 0}=100$.

and the heat and molar fluxes. Since it is these fluxes that determine the variation of the primary variables, we also want to isolate the thermodynamic effects upon these fluxes and explore their variation with the initial heptane temperature.

\subsubsection{First-order statistics}

The quantities of interest can be categorized into the primary variables, the thermodynamic variables, the transport properties, and finally the fluxes. For each of these quantities, we present mean quantities that are calculated by either averaging over the streamwise direction for two-dimensional layers, or over homogeneous $\left(x_{1}, x_{3}\right)$-planes for the three-dimensional layer.

The mean primary variables $\left(T, \rho, Y_{h}\right.$ and $\left.p\right)$ illustrated in figure $10(a-d)$ for Runs 1-8 show a closeness of the averaged profiles for Runs 1-6 and Run 8 for all the variables except the pressure. It is only for the largest constant $\alpha_{B K}$ that the density and temperature exhibit a small peak on the nitrogen side of the layer, while the temperature and the heptane mass fraction respectively display a local maximum and a local minimum at the site of the initial boundary between the two fluids. A larger initial temperature in the heptane stream induces an accordingly lower density and a more gas-like behaviour. The pressure is the only quantity which shows more important variations with the identity and value of the thermal diffusion factors, and the local pressure minimum displayed in the layer is the direct consequence of the strong pairing. 

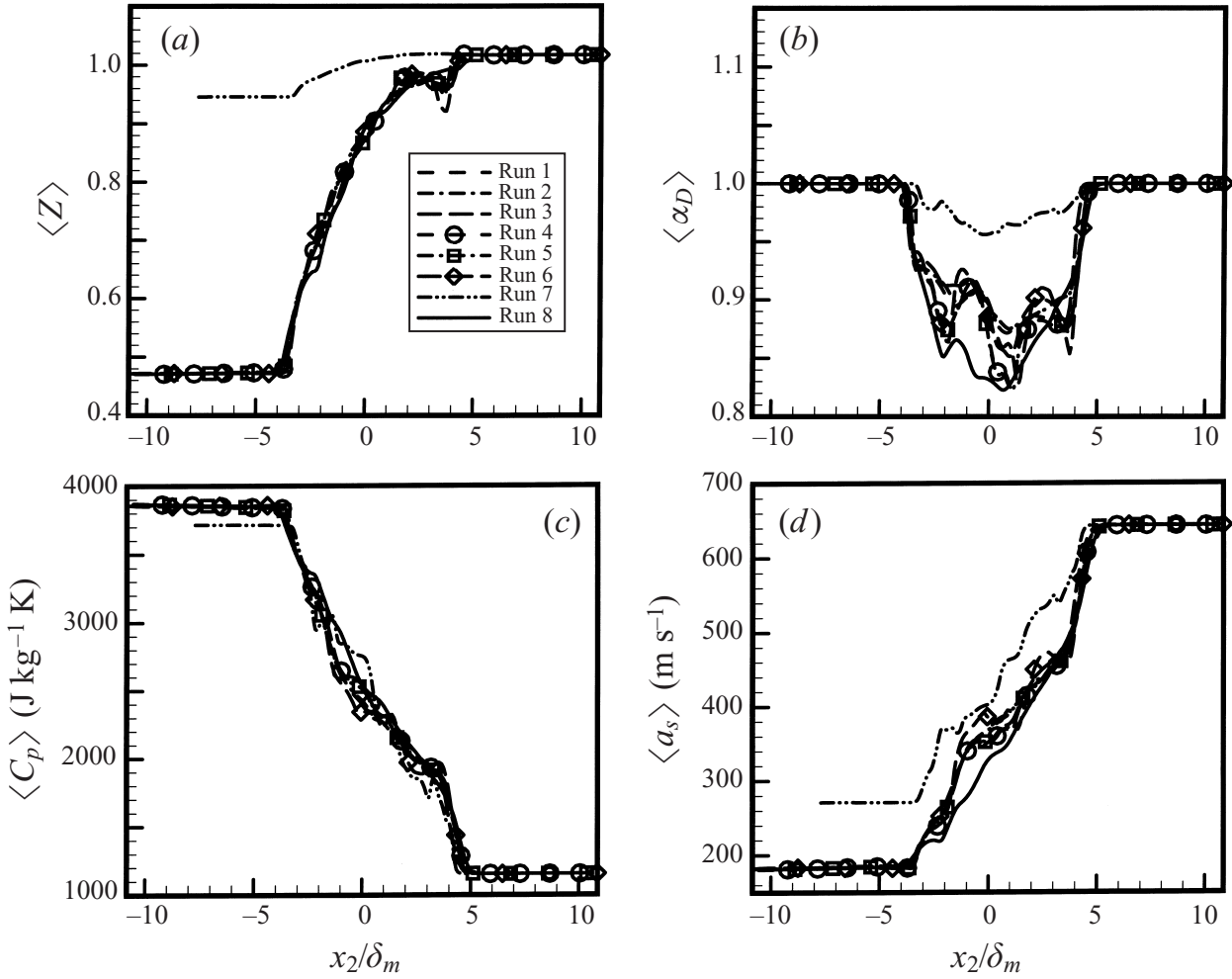

FiguRE 11. Mean thermodynamic quantities versus a non-dimensional cross-stream variable: compression factor $(a)$, thermal diffusion factor $(b)$, heat capacity at constant pressure $(c)$, and speed of sound $(d)$ for Runs $1-8$ at the non-dimensional time $t \Delta U_{0} / \delta_{\omega, 0}=100$.

With the exception of Run 7 which displays a closer resemblance to the wellknown atmospheric conditions, most of the thermodynamic quantities, $Z, \alpha_{D}, C_{p}$ and $a_{s}$ shown in figure $11(a-d)$ have a similar average variation for all runs; an exception is Run 6 for which $Z$ exhibits a local minimum at the nitrogen edge of the layer and for which the mixture has the largest departure from ideality. In agreement with the flow visualizations, most mixture non-ideality for the $\alpha_{B K}$-constant runs is found at the edges and in the innermost region of the mixing layer.

To examine the peculiarities of the transport coefficients under supercritical conditions, mean profiles of $\alpha_{I K}, \alpha_{B K}$ and $\lambda_{I K}^{\prime} / \lambda$ are shown in figures $12(a)$ to $12(c)$ respectively. The variation of $\alpha_{B K}$ at prescribed, constant $\alpha_{I K}$ and that of $\alpha_{B K}$ at prescribed, constant $\alpha_{B K}$ is entirely the result of (2.21) which states that the difference of the two thermal diffusion factors is a thermodynamic function. Since this function is particular to the species under consideration, the following discussion applies only to the heptane-nitrogen pair at these thermodynamic conditions and cannot be generalized to other species combinations. When $\alpha_{I K}$ is assumed positive and constant, $\alpha_{B K}$ is generally negative and large across the mixing layer (see figure 12b) and therefore $\lambda_{I K}^{\prime} / \lambda<1$. For a pure substance $\lambda_{I K}^{\prime} / \lambda=1$; however, for mixtures this is no longer the case as consistently shown in figure $12(c)$ : it is precisely in the mixing region that the excursions from unity occur and they are enhanced by larger values of the assumed constant $\alpha_{B K}$. Since for prescribed constant $\alpha_{I K}$ figure $10(a)$ shows that $\alpha_{B K}$ is generally negative and large, the associated term in (2.18) has a contribution to the molar flux that has the same sign as the mean temperature gradient (which is positive) while the 

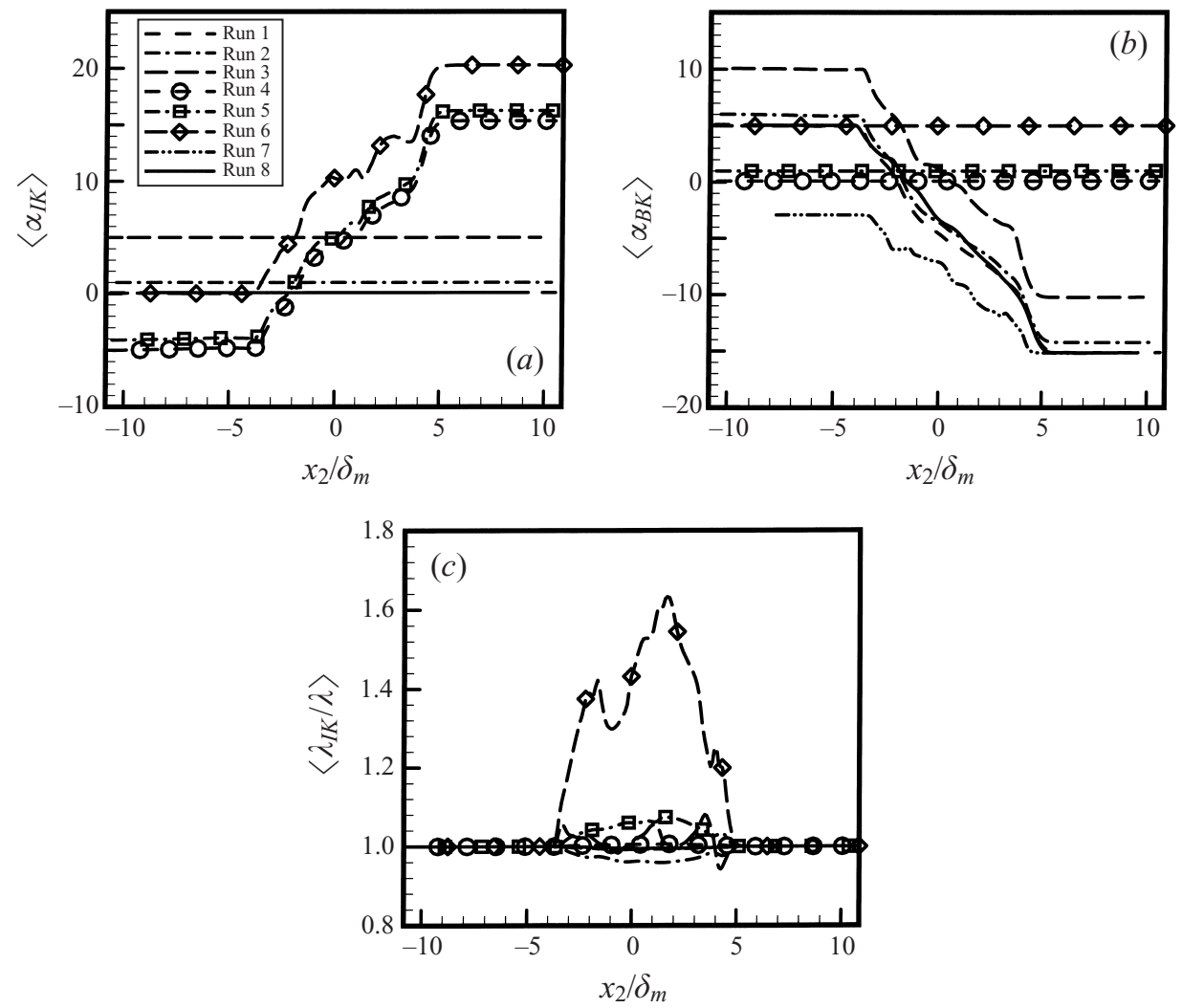

FiguRE 12. Mean transport coefficients versus a non-dimensional cross-stream variable: IrwingKirkwood thermal diffusion factor $(a)$, Bearman-Kirkwood thermal diffusion factor $(b)$, and thermal conductivity $(c)$ for Runs $1-8$ at the non-dimensional time $t \Delta U_{0} / \delta_{\omega, 0}=100$.

term associated with the mean mass fraction gradient is positive (because the gradient is negative); this means that the Soret term effectively increases the Fickian term, as shown on figure 13(a) for the smallest $\alpha_{I K}$ value. In fact, the plots in figure 13(a) show that the molar flux vector is on average aligned with the Fickian term and is enhanced by the Soret effect, while the pressure contribution is small (as also found in the previous fluid drops study of Harstad \& Bellan 2000). In the same manner, the heat flux has a positive contribution from the Dufour term in $\boldsymbol{J}^{\prime}$, and a negative contribution from the thermal conductivity term resulting in a vector magnitude dominated by the Fourier term (see figure 13b) with the pressure and Dufour contributions being negligible. As the value of $\alpha_{I K}$ increases, all these effects become exacerbated.

A completely different picture emerges from the assumed constant $\alpha_{B K}$ simulations. Under these conditions, $\alpha_{I K}$ is generally positive and large across the mixing layer, implying that $\lambda_{I K}^{\prime} / \lambda>1$ as clearly seen in figure $12(c)$. Similar arguments to those above lead to the result that the Soret term is negligible (see figure 13c), the molar flux being essentially represented by the Fickian contribution, and that the Dufour term is significant, generally anti-aligned with the Fourier term and thus reduces the heat flux (see figure 13d). The pressure contribution is again found to be unimportant.

These considerations indicate that accurate predictions of fluid flow behaviour must necessarily rely upon the accurate knowledge of the value of the thermal diffusion factors. 

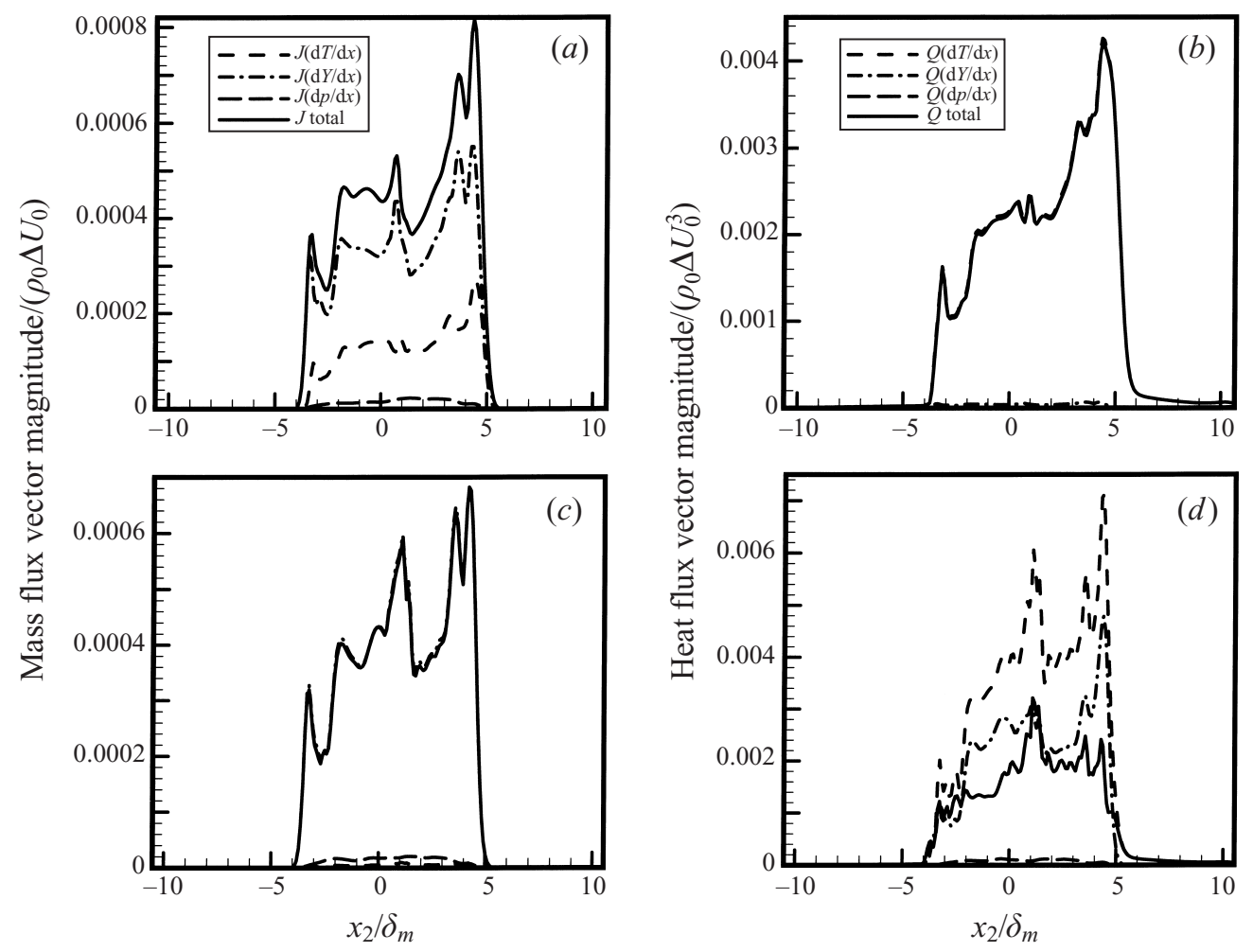

FiguRE 13. Mean mass flux $(a, c)$ and heat flux $(b, d)$ vector magnitudes versus a non-dimensional cross-stream variable for Run $1(a, b)$ and Run $4(c, d)$ at the non-dimensional time $t \Delta U_{0} / \delta_{\omega, 0}=100$. The total heat and the molar flux ( $Q$ total and $J$ total, respectively) are considered to be sums of three terms proportional to the temperature gradient $[Q(\mathrm{~d} T / \mathrm{d} x)$ and $J(\mathrm{~d} T / \mathrm{d} x)]$, mass fraction gradient $[Q(\mathrm{~d} Y / \mathrm{d} x)$ and $J(\mathrm{~d} Y / \mathrm{d} x)]$ and pressure gradient $[Q(\mathrm{~d} p / \mathrm{d} x)$ and $J(\mathrm{~d} p / \mathrm{d} x)]$.

\subsubsection{Second-order statistics}

The above discussion primarily identified the differences in behaviour between assumed constant- $\alpha_{I K}$ and assumed constant- $\alpha_{B K}$ two-dimensional mixing layers, and related the difficulty of achieving transition in the strongly density stratified flow. However, none of the above results enables us to quantitatively assess the final state of the fluctuations created during the evolution of the layer and how they relate to the discussed mean quantities. Second-order statistics are ideally suited for exploring the differences in the final state of the layer due to the evolved fluctuations in the dynamic and thermodynamic quantities. Therefore, here we devote attention to the evolution of surface (for two-dimensions) or volume (for three-dimensions) averages of the relative positive fluctuations of the spanwise vorticity to corroborate our previous findings related to compressibility effects in two-dimensions; and to vortex stretching, creation of small scales and turbulence transition in three-dimensions. We also investigate the kinetic energy profile at the final simulation time in order to assess the energy distribution in the layer, and finally we examine the distribution of the correlations of the gradients of temperature and mass fraction fluctuations at the final time as a measure of Soret and Dufour effects.

Shown in figure 14 is the root mean square of the positive fluctuations of the spanwise vorticity as a function of the non-dimensional time ( $H$ is the Heaviside function). 


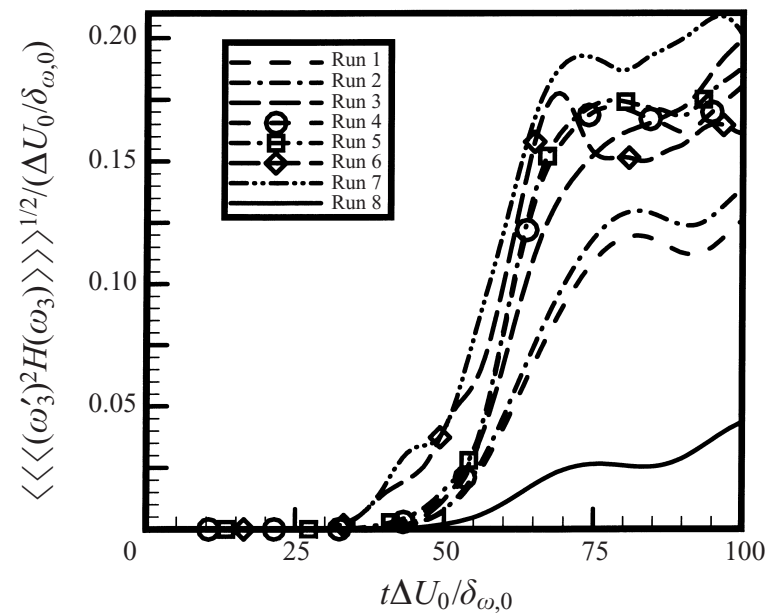

FIGURE 14. Evolution of the averaged relative positive fluctuations of the spanwise vorticity for Runs 1-8.

Consistent with the final time flow visualization, there is no sudden emergence of small scales in the three-dimensional layer; as mentioned above, the sudden increase noticeable in the two-dimensional layers is not indicative of turbulence initiation, but of $\nabla \cdot \boldsymbol{u}$ (i.e. local) compressibility and density gradient effects. Since $\boldsymbol{\nabla} \cdot \boldsymbol{u}$ contains both $M_{c}$ effects (through the isentropic compressibility) and dissipation effects, examination of $M_{c}$ contours (not shown) is indicative of how $M_{c}$ compressibility affects $\nabla \cdot \boldsymbol{u}$ and eventually the vorticity. Indeed, Mach number contours of constant- $\alpha_{B K}$ runs feature higher values than those of constant- $\alpha_{I K}$ runs, consistent with the figure 14 higher vorticity for the constant- $\alpha_{B K}$ cases.

The mean kinetic energy, $K=0.5\left\langle u_{i}^{\prime} u_{i}^{\prime}\right\rangle$, depicted in figure 15 at the final simulation time reveals a maximum in the nitrogen side of the mixing layers indicating that it is there that most fluctuations occur; for the three-dimensional mixing layer the location of the maximum is shifted further towards the nitrogen side. The larger kinetic energy for the larger $T_{2}$ mixing layer is a consequence of the small momentum ratio of the two streams which makes it easier to entrain and to create the small-scale structure.

The ultimate explanation for the dissimilarity between assumed constant- $\alpha_{I K}$ and assumed constant $\alpha_{B K}$ two-dimensional mixing layers is apparent in figure 16 where the distribution of the single and mixed correlations of the gradients of temperature and mass fraction fluctuations at the final time are illustrated. The striking feature of these correlations is the strong fluctuations excited when $\alpha_{B K}$ is assumed constant and large, whose magnitude decreases with that of $\alpha_{B K}$. These fluctuations are related to the dissipation and act to reduce the scalar temporal variance, which explains the reduced growth of the layer, the increased growth with increasing $T_{2}$ and the inability of the layer to reach transition. An important aspect of these correlations is that except for the large $T_{2}$ case, $\left\langle\nabla T^{\prime} \nabla Y_{h}^{\prime}\right\rangle \leqslant 0$ indicating that for large constant $\alpha_{B K}$ (and consequently for large positive $\alpha_{I K}$ ) each of the Soret and Dufour terms is strongly negatively correlated at large initial temperature ratios between the two streams; in fact, the only instance when these cross-transport terms are positively correlated is when $T_{2}$ is larger, indicating that the memory of the initial conditions is lost. These strong correlations are a direct result of the strong cross-coupling; if they were absent, there would be strong fluctuations in the heat flux which are unphysical because diffusion processes smear profiles at the molecular level. Alternatively, one 


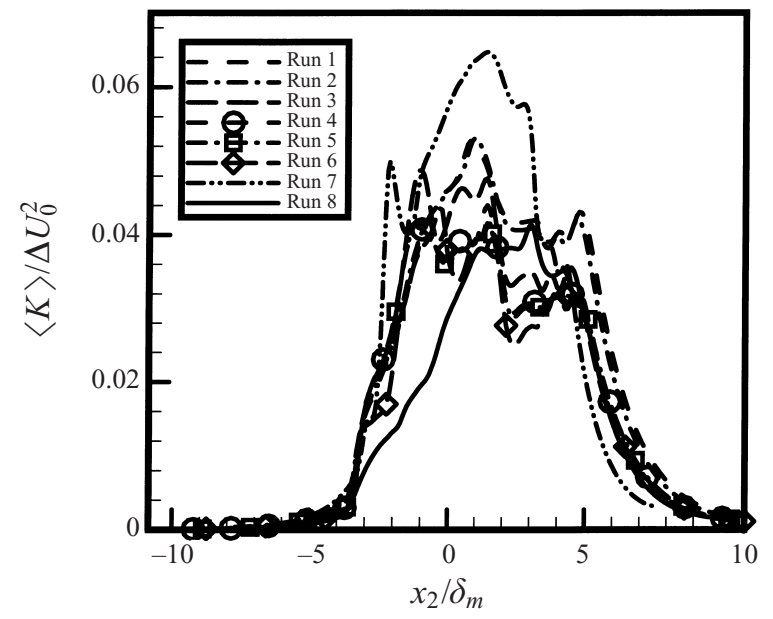

FIGURE 15. Kinetic energy versus a non-dimensional cross-stream variable at the non-dimensional time $t \Delta U_{0} / \delta_{\omega, 0}=100$.

may state that since the fluctuations of the heat flux vector are necessarily limited owing to the diffusional processes, strong cross-term coupling through fixed positive $\alpha_{B K}$ implies that strong correlations must occur. These findings explain why $\alpha_{B K}$ influences mixing whereas $\alpha_{I K}$ influences entrainment: $\alpha_{B K}$ determines the diffusional cross-term and therefore influences the diffusional mixing; $\alpha_{I K}$ determines the heat flux cross-term and thus more dramatically influences the enthalpy, temperature and density profiles, as well as the amounts of shear and vorticity.

\section{Summary and conclusions}

A direct numerical simulation of a supercritical mixing layer has been conducted using a system of governing equations including the continuity, the momentum, the energy and the species conservation equations, and an equation of state for real gases. This equation of state was based on pure species reference states accurate within $1 \%$ of more highly accurate equations of state over the range of variables used in this study. The diffusional fluxes are mathematically described according to fluctuationdissipation theory which has been used in a previously validated model, and include Soret and Dufour terms along with the well-known Fickian and Fourier terms that dominate at low pressures. Moreover, a term proportional to the pressure gradient appears as well, but the results of the simulations show this term to be negligible over the entire range of parameters studied.

Special attention has also been devoted to the appropriate calculation of transport coefficients, with the exception of the reference viscosity which is set by the initial value of the Reynolds number. The diffusivity and thermal conductivity values are computed from calculated values of the Schmidt and Prandtl numbers through expressions which are approximate fits to the correct values as functions of the mass fraction. Owing to the dual possibility in mathematically formulating the heat flux vector as shown by Harstad \& Bellan (2000), several possibilities are available for defining the thermal conductivity; however, only one of these converges to the kinetic theory definition. Some of our results show the ratio between the flux defined and the correct definition of the thermal conductivity.

Similar to the thermal conductivity definition, there are also two possibilities for 

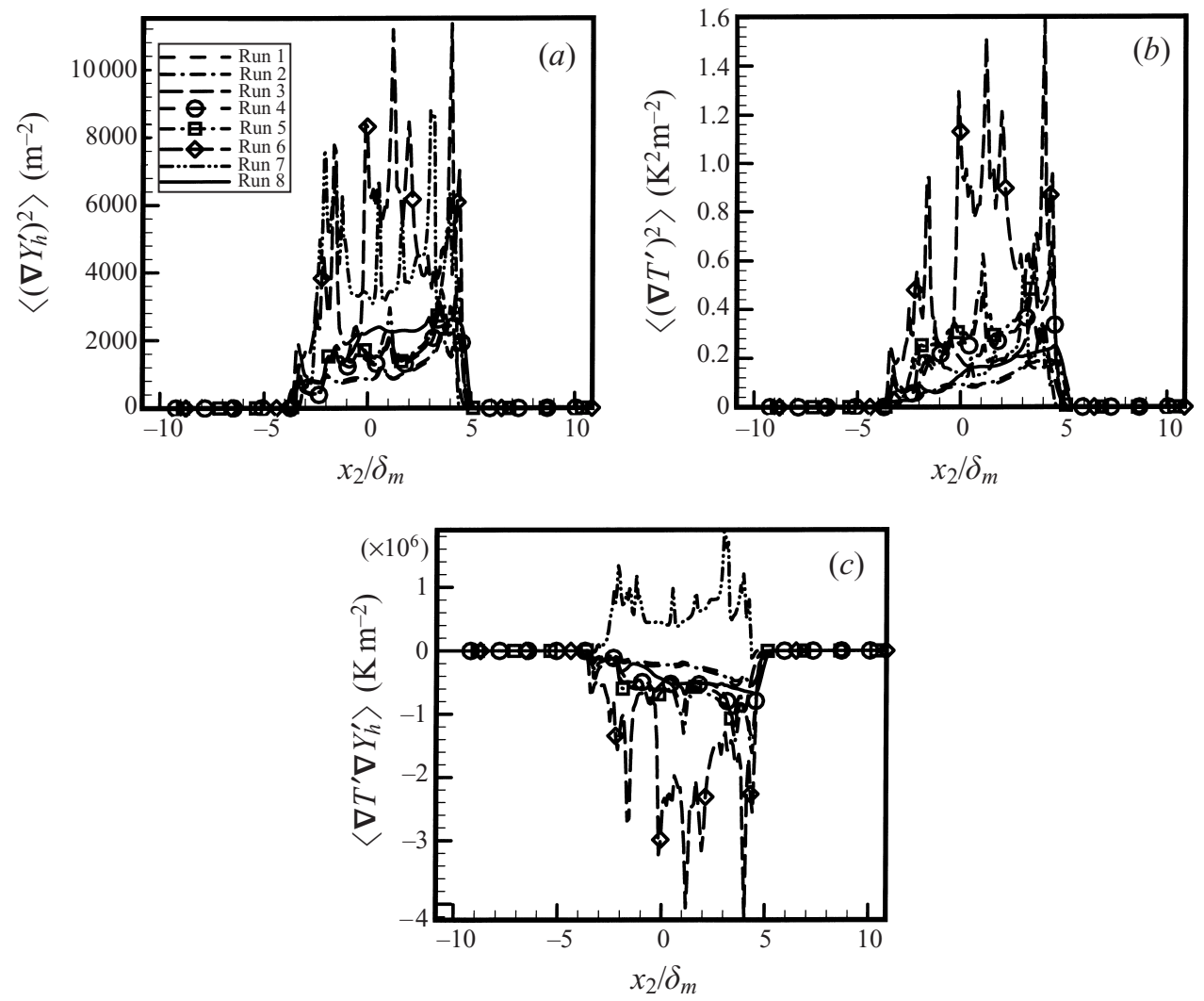

FIGURE 16. Cross-stream distribution of the single and mixed correlations of the gradients of temperature and mass fraction fluctuations at the non-dimensional time $t \Delta U_{0} / \delta_{\omega, 0}=100:\left\langle\left(\nabla Y_{h}^{\prime}\right)^{2}\right\rangle$ in $(a) ;\left\langle\left(\nabla T^{\prime}\right)^{2}\right\rangle$ in $(b) ;\left\langle\nabla Y_{h}^{\prime} \nabla T^{\prime}\right\rangle$ in $(c)$.

defining the thermal diffusion factor, and their difference is a thermodynamic function as shown by Harstad \& Bellan (2000). In general, the magnitude of these thermal diffusion factors is not well documented, and therefore part of our study explores the differences between the predictions obtained by setting constant and positive each of the defined thermal diffusion factors.

The configuration studied is that of a temporal, forced two-dimensional or threedimensional mixing layer where the hydrocarbon is in the lower stream and is initially at a lower temperature than the upper nitrogen stream. Although the approximate value of the thermal diffusion factor for heptane-nitrogen was determined from the previous study of Harstad \& Bellan (2000), since such values are not generally available, two-dimensional mixing layers are used to study the influence of the assumed constant thermal diffusion factors. Additionally, the influence of the larger initial temperature of the hydrocarbon stream is studied as well. The three-dimensional mixing layer results are used to evaluate the influence of the density stratification on transition to turbulence (which has not been achieved) in the absence of gravitational effects.

Our conclusions reveal the different impact of the two forms of thermal diffusion factors, the effect of the mass diffusion factor and that of the compression factor on the entrainment and growth of the layer.

The results show that when assumed constant, one of the thermal diffusion factors is intimately related to species mixing, whereas the other is intimately related to 
entrainment. In all cases, mixing is found to be entrainment controlled as inherently the two-dimensional mixing layers do not develop turbulent features owing to lack of vortex stretching, and as the three-dimensional layer mixing is inhibited by the large momentum change necessary to entrain the lower stream. This results in minimal formation of small turbulent scales as indicated by both flow visualizations and timewise evolution of relative positive spanwise vorticity fluctuations. Augmentation of the initial temperature of the hydrocarbon stream decreases the density stratification, resulting in increased turbulent kinetic energy and increased layer growth.

Quantification of the thermodynamic state of the mixture shows that the mixture exhibits strong departure both from a perfect gas and from mixture ideality. In fact, the departure from mixture ideality reduces diffusional (molecular) mixing and counteracts the effects of one form of assumed constant thermal diffusion factor.

For the supercritical temporal mixing layers examined here we have also identified a secondary mechanism (the primary one being the initial density stratification) that may hinder transition to turbulence. The formation of very sharp local density gradients induces additional stratification, and since density interfaces were found in previous studies to hinder entrainment and transfer of energy from large- to smallscale eddies, this mechanism may suppress transition. This suppression is amplified by the effect of mixture non-ideality which reduces molecular mixing.

Finally, the completely different roles of the two forms of thermal diffusion factors, one promoting density gradients, shear and vorticity, and the other promoting diffusional mixing, imply that accurate predictions of shear layer evolution is contingent upon the knowledge of the value of these cross-term transport coefficients. This conclusion has special consequences if direct numerical simulations of supercritical mixing layers are intended to be used as databases for the development of predictive turbulence models.

This research was conducted at the California Institute of Technology's Jet Propulsion Laboratory (JPL) while the first author was a Caltech Postdoctoral Scholar. Joint sponsorship was provided by General Electric (GE) through the Air Force Office of Scientific Research (AFOSR) Focused Research Initiative program with Dr Hukam Mongia from GE serving as contract monitor and by the National Aeronautics and Space Administration, the John Glenn Research Center at Lewis Field with Dr Daniel L. Bulzan as technical contract monitor. Computational resources were provided by the supercomputing facility at JPL. The constructive comments of one of the reviewers are acknowledged, prompting us to explore in more detail the various contributions to vorticity and vorticity magnitude production. The authors also wish to thank the Associate Editor, Professor S. K. Lele, for pertinent comments.

\section{Appendix A}

The mixing rules recommended by Harstad et al. (1997) are

$$
\begin{gathered}
A_{\alpha \beta}=0.457236\left(R_{u} T_{c, \alpha \beta}\right)^{2}\left[1+C_{\alpha \beta}\left(1-\sqrt{T / T_{c, \alpha \beta}}\right)\right]^{2} / p_{c, \alpha \beta}, \\
B_{\alpha}=0.077796 R_{u} T_{c, \alpha \alpha} / p_{c, \alpha \alpha}, \\
C_{\alpha \beta}=0.37464+1.54226 \Omega_{\alpha \beta}-0.26992 \Omega_{\alpha \beta}^{2},
\end{gathered}
$$

where the subscript $c$ refers to critical properties (i.e. $T / T_{c}$ is the reduced temperature). The diagonal elements of the 'critical' matrices are equal to their pure substance 
counterparts, i.e. $T_{c, \alpha \alpha}=T_{c, \alpha}, p_{c, \alpha \alpha}=p_{c, \alpha}$, and $\Omega_{\alpha \alpha}=\Omega_{\alpha}$ where $\Omega$ is the species acentric factor and the pure substance critical properties for heptane and nitrogen are given in table 1 . The off-diagonal elements are evaluated through additional rules

$$
\begin{gathered}
T_{c, \alpha \beta}=\sqrt{T_{c, \alpha \alpha} T_{c, \beta \beta}}\left(1-k_{\alpha \beta}\right), \quad p_{c, \alpha \beta}=Z_{c, \alpha \beta}\left(R_{u} T_{c, \alpha \beta} / v_{c, \alpha \beta}\right), \\
v_{c, \alpha \beta}=\frac{1}{8}\left[\left(v_{c, \alpha \alpha}\right)^{1 / 3}+\left(v_{c, \beta \beta}\right)^{1 / 3}\right]^{3}, \quad Z_{c, \alpha \beta}=\frac{1}{2}\left(Z_{c, \alpha \alpha}+Z_{c, \beta \beta}\right), \quad \Omega_{\alpha \beta}=\frac{1}{2}\left(\Omega_{\alpha \alpha}+\Omega_{\beta \beta}\right),
\end{gathered}
$$

where the diagonal elements of each of the above symmetric matrices are also equal to the pure substance values and $k_{\alpha \beta}$ is the binary interaction parameter which is a function of the species being considered and is taken to be $k_{\alpha \beta}=0.1$ for $\alpha \neq \beta$ and zero otherwise for heptane-nitrogen.

\section{Appendix B}

Miscellaneous thermodynamic relations are

$$
\begin{gathered}
\frac{\partial A_{m}}{\partial X_{\alpha}}=2 \sum_{\beta} A_{\alpha \beta} X_{\beta}, \\
\frac{\partial A_{m}}{\partial T}=-\frac{1}{T} \sum_{\alpha} \sum_{\beta} X_{\alpha} X_{\beta} A_{\alpha \beta} G_{\alpha \beta}, \\
\frac{\partial^{2} A_{m}}{\partial X_{\alpha} \partial T}=\frac{-2}{T} \sum_{\beta} A_{\alpha \beta} G_{\alpha \beta} X_{\beta}, \\
\frac{\partial^{2} A_{m}}{\partial T^{2}}=\frac{0.457236 R_{u}^{2}}{2 T} \sum_{\alpha} \sum_{\beta} X_{\alpha} X_{\beta} C_{\alpha \beta}\left(1+C_{\alpha \beta}\right) \frac{T_{c, \alpha \beta}}{p_{c, \alpha \beta}} \sqrt{\frac{T_{c, \alpha \beta}}{T}},
\end{gathered}
$$

where

$$
G_{\alpha \beta}=\frac{C_{\alpha \beta} \sqrt{T / T_{c, \alpha \beta}}}{1+C_{\alpha \beta}\left(1-\sqrt{T / T_{c, \alpha \beta}}\right)}
$$

\section{REFERENCES}

Atsavapranee, P. \& Gharib, M. 1997 Structures in stratified plane mixing layers and the effect of cross shear. J. Fluid Mech. 342, 53-86.

Bird, R. B., Stewart, W. E. \& Lightfoot, E. N. 1960 Transport Phenomena. John Wiley and Sons.

Briggs, D. A., Ferziger, J. H., Koseff, J. R. \& Monismith, S. G. 1998 Turbulent mixing in a shear-free stably stratified two-layer fluid. J. Fluid Mech. 354, 175-208.

Brown, G. L. \& Roshko, A. 1974 On density effects and large structure in turbulent mixing layers. J. Fluid Mech. 64, 775-816.

Chapman, S. \& Cowling, T. G. 1970 The Mathematical Theory of Nonuniform Gases. Cambridge University Press.

Chenroudi, B., TAlley, D. \& Coy, E. 1999 Initial growth rate and visual characteristics of a round jet into a sub- to super-critical environment of relevance to rocket, gas turbine and diesel engines. AIAA Paper 99-0206.

Cortesi, A. B., Smith, B. L., Yadigaroglu, G. \& Banerjee, S. 1999 Numerical investigation of the entrainment and mixing processes in neutral and stably-stratified mixing layers. Phys. Fluids 11, 162-185.

Cortesi, A. B., Yadigaroglu, G. \& Banerjee, S. 1998 Numerical investigation of the formation of three-dimensional structures in a stably-stratified mixing layer. Phys. Fluids 10, 1449-1473.

Cussler, E. L. 1984 Diffusion, Mass Transfer in Fluid Systems. Cambridge University Press. 
Dimotakis, P. 1986 Two-dimensional shear layer entrainment. AIAA J. 24, 1791-1796.

Drazin, P. G. \& ReID, W. H. 1981 Hydrodynamic Stability. Cambridge University Press.

Gerz, T., Schumann, U. \& Elghobashi, S. E. 1989 Direct numerical simulation of stratified homogeneous turbulent shear flows. J. Fluid Mech. 200, 563-594.

Hannoun, I. A., Fernando, H. J. S. \& List, E. J. 1988 Turbulence structure near a sharp density interface. J. Fluid Mech. 189, 189-209.

Harstad, K. \& Bellan, J. 1998 Isolated fluid oxygen drop behavior in fluid hydrogen at rocket chamber pressures. Intl J. Heat Mass Transfer 41, 3537-3550.

Harstad, K. \& Bellan, J. 1999 The Lewis number under supercritical conditions. Intl J. Heat Mass Transfer 42, 961-970.

Harstad, K. \& Bellan, J. 2000 An all-pressure fluid-drop model applied to a binary mixture: heptane in nitrogen. Intl J. Multiphase Flow 26, 1675-1706.

Harstad, K. G., Miller, R. S. \& Bellan, J. 1997 Efficient high pressure state equations. A.I.Ch.E. $J .43,1605-1610$.

Hernan, M. A. \& Jimenez, J. 1982 Computer analysis of a high-speed film of the plane mixing layer. J. Fluid Mech. 119, 323-345.

Hirshfelder, J. O., Curtis, C. F. \& Bird, R. B. 1964 Molecular Theory of Gases and Liquids. John Wiley and Sons.

KeIzer, J. 1987 Statistical Thermodynamics of Nonequilibrium Processes. Springer.

Kennedy, C. A. \& CARPEnTER, M. H. 1994 Several new numerical methods for compressible shear-layer simulations. Appl. Numer. Maths 14, 397-433.

Kennedy, C. A. \& CHEN, J. H. 1998 Mean flow effects on the linear stability of compressible planar jets. Phys. Fluids 10, 615-626.

Kozusko, F., Grosch, C. E., Jackson, T. L., Kennedy, C. A. \& Gatski, T. B. $1996 a$ The structure of variable property, compressible mixing layers in binary gas mixtures. Phys. Fluids 8, 1945-1953.

Kozusko, F., Lasseigne, D. G., Grosch, C. E. \& JaCKson, T. L. $1996 b$ The stability of compressible mixing layers in binary gases. Phys. Fluids 8, 1954-1963.

Lu, G. \& LELE, S. K. 1994 On the density ratio effect on the growth rate of a compressible mixing layer. Phys. Fluids 6, 1073-1075.

Maslowe, S. A. \& Kelly, R. E. 1971 Inviscid instability of an unbounded heterogeneous shear layer. J. Fluid Mech. 48, 405-415.

Mayer, W., Ivancic, B., Schik, A. \& Hornung, U. 1998 Propellant atomization in LOX/GH2 rocket combustors. AIAA Paper 98-3685.

Mayer, W., Schik, A., Schweitzer, C. \& Schaffler, M. 1996 Injection and mixing processes in high pressure LOX/GH2 rocket combustors. AIAA Paper 96-2620.

Miller, R. S. \& Bellan, J. 1999 Direct numerical simulation of a confined three-dimensional gas mixing layer with one evaporating hydrocarbon-droplet laden stream. J. Fluid Mech. 384, 293-338.

Miller, R. S. \& Bellan, J. 2000 Direct numerical simulation and subgrid analysis of a transitional droplet laden mixing layer. Phys. Fluids 12, 650-671.

Moser, R. D. \& Rogers, M. M. 1991 Mixing transition and the cascade to small scales in a plane mixing layer. Phys. Fluids A 3, 1128-1134.

Oefelein, J. C. \& YANG, V. 1996 Analysis of transcritical spray phenomena in turbulent mixing layers. AIAA Paper 96-0085.

OKong'o, N. \& Bellan, J. 2000 Entropy production of emerging turbulent scales in a temporal supercritical n-heptane/nitrogen three-dimensional mixing layer. Proc. Combust. Inst. 28, 497504.

Papamoschou, D. \& Roshko, A. 1988 The compressible turbulent shear layer: an experimental study. J. Fluid Mech. 197, 453-477.

Poinsot, T. J. \& Lele, S. K. 1992 Boundary conditions for direct numerical simulations of compressible viscous flows. J. Comput. Phys. 101, 104-129.

Prausnitz, J., Lichtenthaler, R. \& De Azevedo, E. 1986 Molecular Thermodynamics for FluidPhase Equilibrium. Prentice-Hall.

Reid, R. C., Prausnitz, J. M. \& Polling, B. E. 1987 The Properties of Gases and Liquids, 4th Edn. McGraw-Hill.

SARMAn, S. \& Evans, D. J. 1992 Heat flux and mass diffusion in binary Lennard-Jones mixtures. Phys. Rev. A 45, 2370-2379. 